\title{
A New Synthetic Method for $\alpha$-Alkoxycarbonyl Iminium Salt and Its Reaction with Nucleophiles
}

\author{
Makoto Shimizu, ${ }^{*}$ Hiroyuki Itou and Megumi Miura \\ Department of Chemistry for Materials, Mie University, Tsu, Mie 514-8507, Japan
}

\begin{abstract}
General Aspects
Infrared spectra were determined on a JASCO IR-810 spectrometer. ${ }^{1} \mathrm{H}$ NMR and ${ }^{13} \mathrm{C}$ NMR spectra were recorded with a JEOL EX-270 or a JEOL a -500 spectrometer using tetramethylsilane as an internal standard. Mass spectra were recorded on a Mariner TK3500 spectrometer. Ether and THF were distilled from benzophenone ketyl immediately before use. $\mathrm{CH}_{2} \mathrm{Cl}_{2}$ was distilled from calcium hydride and stored over Molecular Sieves 4A. DME was distilled from calcium hydride and then copper(1) chloride and stored over sodium. Purification of products was performed by column chromatography on silica gel (Merck Silica Gel-60) and/or preparative TLC on silica gel (Merck Kiesel Gel PF254).
\end{abstract}

\section{Ethyl 2-cyano-2-(dibenzylamino)acetate (4)}

Typical procedure (Table 1, entry 9): Under an argon atmosphere, 1-ethoxy-2-dibenzylamino-1-trimethylsiloxyethylene (0.30 ml, $0.15 \mathrm{mmol}, 0.5 \mathrm{M}$ in $\mathrm{CH}_{2} \mathrm{Cl}_{2}$ ) and diethyl aluminium cyanide $(0.30 \mathrm{mmol}, 0.30 \mathrm{ml}, 1.0 \mathrm{M}$ in toluene) were added to a solution of 2,3-dichloro-5,6-dicyano-1,4-benzoquinone (DDQ) (35.1 mg, $0.15 \mathrm{mmol}$ ) in DME (0.5 ml) at $-50{ }^{\circ} \mathrm{C}$. The reaction mixture was allowed to warm to ambient temperature with stirring for $11.5 \mathrm{~h}$. The reaction was quenched with $10 \% \mathrm{aqNa}_{2} \mathrm{SO}_{3}$, and the whole mixture was extracted with ethyl acetate $(10 \mathrm{ml} \times 3)$. The combined extracts were washed with brine, dried over anhydrous $\mathrm{Na}_{2} \mathrm{SO}_{4}$, and concentrated in vacuo. The crude product was purified by preparative TLC on silica gel (developed once with EtOAc/ $n$-hexane $=1 / 7$ and then with EtOAc/toluene $=$ 1/50) to give ethyl 2-cyano-2-(dibenzylamino)acetate (71\%, $32.7 \mathrm{mg})$.

- Ethyl 2-cyano-2-(dibenzylamino)acetate (4): colorless oil; ${ }^{1} \mathrm{HNMR}\left(\mathrm{CDCl}_{3}\right) \delta 1.31$ (t, $J=7.3 \mathrm{~Hz}, 3 \mathrm{H}$ ), 3.57 (d, $J=13.5 \mathrm{~Hz}, 2 \mathrm{H}$ ), 3.89 (d, $J=13.5 \mathrm{~Hz}, 2 \mathrm{H}), 4.22$ (q, $J=7.3 \mathrm{~Hz}, 2 \mathrm{H}$ ), 4.39 (s, $1 \mathrm{H}), 7.26-7.45$ (m, 10H); ${ }^{13} \mathrm{CNMR}\left(\mathrm{CDCl}_{3}\right) \delta \quad$ 14.0, 55.9, 56.6, 63.0, 113.0, 127.9, 128.6, 128.9, 136.9, 164.6; IR (neat) 3020, 2845, 1760, 1453, 1215, 1019, 755, $670 \mathrm{~cm}^{-1}$; HRMS (ESI): Calculated for $\mathrm{C}_{19} \mathrm{H}_{20} \mathrm{~N}_{2} \mathrm{O}_{2}(\mathrm{M}+\mathrm{H})^{+}$. 309.15, found 309.04. 


\section{Ethyl 2-(dibenzylamino)butanoate (5b)}

Typical procedure (Table 2, entry 2): Under an argon atmosphere, a solution of 1-ethoxy-2-dibenzylamino-1-trimethylsiloxyethylene (0.30 ml, $0.15 \mathrm{mmol}, 0.5 \mathrm{M}$ in $\mathrm{CH}_{2} \mathrm{Cl}_{2}$ ) and ethylmagunesium bromide $(0.31 \mathrm{ml}, 0.30 \mathrm{mmol}, 0.98 \mathrm{M}$ in THF $)$ were added to a solution of DDQ (35.1 mg, $0.15 \mathrm{mmol})$ in DME $(0.5 \mathrm{ml})$ at $-55{ }^{\circ} \mathrm{C}$. The reaction mixture was allowed to warm to ambient temperature with stirring for $15 \mathrm{~h}$. The reaction was quenched with sat aqNaHCO 3 , and the whole mixture was extracted with ethyl acetate (10 $\mathrm{ml} \mathrm{x} \mathrm{3).} \mathrm{The} \mathrm{combined}$ extracts were washed with brine, dried over anhydrous $\mathrm{Na}_{2} \mathrm{SO}_{4}$, and concentrated in vacuo. The crude product was purified by preparative TLC on silica gel $($ EtOAc/toluene $=1 / 50)$ to give ethyl 2-(dibenzylamino)butanoate (66\%, $30.8 \mathrm{mg})$.

\section{Ethyl 2-(dibenzylamino)propanoate (5a)}

colorless oil; HNMR $\left(\mathrm{CDCl}_{3}\right) \delta 1.33$ (t, $\left.J=13.4 \mathrm{~Hz}, 6 \mathrm{H}\right), 3.49$ (q, $\left.J=7.3 \mathrm{~Hz}, 1 \mathrm{H}\right), 3.66$ (d, $J=14.0 \mathrm{~Hz}, 2 \mathrm{H}), 3.81$ (d, $J=14.0 \mathrm{~Hz}, 2 \mathrm{H}), 4.14-4.27$ (m, 2H), 7.21-7.39 (m, 10H); ${ }^{13} \mathrm{CNMR}$ $\left(\mathrm{CDCl}_{3}\right) \delta$ 14.5, 15.0, 54.4, 56.1, 60.1, 126.9, 128.2, 128.6, 140.0, 173.7; IR (neat) 3062, 3028, 2981, 2936, 2842, 2803, 1950, 1874, 1729, 1602, 1493, 1452, 1373, 1328, 1244, 1196, 1146, 1061, 1026, 745, $699 \mathrm{~cm}^{-1}$; HRMS (ESI): Calculated for $\mathrm{C}_{20} \mathrm{H}_{25} \mathrm{NO}_{2}(\mathrm{M}+\mathrm{H})^{+}$298.17, found 298.14 .

\section{Ethyl 2-(dibenzylamino)butanoate (5b)}

colorless oil; ${ }^{1} \mathrm{HNMR}\left(\mathrm{CDCl}_{3}\right) \delta 0.91$ (t, $\left.J=7.3 \mathrm{~Hz}, 3 \mathrm{H}\right), 1.34$ (t, $\left.J=7.3 \mathrm{~Hz}, 3 \mathrm{H}\right), 1.67-1.81$ (m, 2H), 3.21 (t, $J=7.6 \mathrm{~Hz}, 1 \mathrm{H}$ ), 3.56 (d, $J=13.9 \mathrm{~Hz}, 2 \mathrm{H}), 3.91$ (d, $J=14.2 \mathrm{~Hz}, 2 \mathrm{H}), 4.15-4.29$ (m, 2H), 7.19-7.38 (m, 10H); ${ }^{13} \mathrm{CNMR}\left(\mathrm{CDCl}_{3}\right) \delta$ 11.0, 14.6, 22.7, 54.5, 59.9, 62.5, 126.9, 128.2, 128.8, 139.8, 173.0; IR (neat) 3023, 2976, 2844, 1721, 1493, 1454, 1373, 1301, 1191, 1146, 1075, 1024, 732, 700, $669 \mathrm{~cm}^{-1}$; HRMS (ESI): Calculated for $\mathrm{C}_{20} \mathrm{H}_{25} \mathrm{NO}_{2}(\mathrm{M}+\mathrm{H})^{+} 312.19$, found 312.35 .

\section{Ethyl 2-(dibenzylamino)pentanoate (5c)}

colorless oil; ${ }^{1} \mathrm{HNMR}\left(\mathrm{CDCl}_{3}\right) \delta \quad 0.79(\mathrm{t}, J=14.7 \mathrm{~Hz}, 3 \mathrm{H}), 1.22-1.31$ (m, 3H), 1.34 (t, $J=15.7$ $\mathrm{Hz}, 3 \mathrm{H}$ ), 1.43-1.51 (m, 1H), 1.63-1.76 (m, 2H), 3.30 (dd, J=8.9 Hz, J=6.1 Hz, 1H), 3.55 (d, $J=14.0 \mathrm{~Hz}, 2 \mathrm{H}$ ), 3.92 (d, J=14.0 Hz, 2H), 4.15-4.29 (m, 2H), 7.21-7.37 (m, 10H); ${ }^{13} \mathrm{CNMR}$ $\left(\mathrm{CDCl}_{3}\right) \delta$ 13.8, 14.5, 19.4, 31.8, 54.5, 60.0, 60.5, 126.9, 128.2, 128.8, 139.8, 173.1; IR (neat) 3032, 2961, 2871, 1719, 1453, 1372, 1186, 1145, 1095, 1027, 751, 750, 699, 674, 465, $417 \mathrm{~cm}^{-1}$; HRMS (ESI): Calculated for $\mathrm{C}_{21} \mathrm{H}_{27} \mathrm{NO}_{2}(\mathrm{M}+\mathrm{H})^{+} 326.20$, found 326.43.

\section{Ethyl 2-(dibenzylamino)-3-phenylpropanoate (5d)}


colorless oil; ${ }^{1} \mathrm{HNMR}\left(\mathrm{CDCl}_{3}\right) \delta 1.29$ (t, $\left.J=7.4 \mathrm{~Hz}, 3 \mathrm{H}\right), 2.96-3.14$ (m, 2H), 3.58 (d, $J=14.0$ Hz, 2H), 3.65 (t, J=15.3 Hz, 1H), 3.94 (d, J=14.0 Hz, 2H), 4.14-4.26 (m, 2H), 7.00-7.26 (m, $15 \mathrm{H}) ;{ }^{13} \mathrm{CNMR}\left(\mathrm{CDCl}_{3}\right) \delta$ 14.5, 35.7, 54.4, 60.2, 62.2, 126.2, 126.9, 128.1,128.2, 128.7, 129.4, 138.2, 139.3, 172.3; IR (neat) 3687, 3025, 1722, 1602, 1494, 1453, 1373, 1248, 1172, 789, 773, 768, 760, 723, 700, 678, 669, 474, $450 \mathrm{~cm}^{-1}$; HRMS (ESI): Calculated for $\mathrm{C}_{25} \mathrm{H}_{27} \mathrm{NO}_{2}$ $(\mathrm{M}+\mathrm{H})^{+}$374.20, found 374.54 .

\section{Ethyl 2-(dibenzylamino)-4-phenylbutanoate (5e)}

colorless oil; ${ }^{1} \mathrm{HNMR}\left(\mathrm{CDCl}_{3}\right) \delta 1.34$ (t, $\left.J=7.3 \mathrm{~Hz}, 3 \mathrm{H}\right), 2.04$ (dd, $J=15.5 \mathrm{~Hz}, J=7.6 \mathrm{~Hz}, 2 \mathrm{H}$ ), 2.41-2.53 (m, 1H), 2.74-2.85 (m, 1H), 3.37 (t, $J=7.3$ Hz, 1H), 3.60 (d, J=13.9 Hz, 2H), 3.93 (d, $J=13.9 \mathrm{~Hz}, 2 \mathrm{H}), 4.22$ (q, $J=7.3 \mathrm{~Hz} 2 \mathrm{H}), 7.01-7.39$ (m, 15H); ${ }^{13} \mathrm{CNMR}\left(\mathrm{CDCl}_{3}\right) \delta \quad 14.8,31.8$, 32.8, 54.9, 60.3, 60.7, 126.0, 127.2, 128.5, 128.6, 129.1, 139.9, 143.1, 173.0; IR (neat) 3064, 3009, 2936, 2853, 1720, 1602, 1494, 1454, 1371, 1252, 1216, 1162, 1074, 1026, 970, 910, 773, 731, 701, 484, 458, 444, 424, $416 \mathrm{~cm}^{-1}$; HRMS (ESI): Calculated for $\mathrm{C}_{26} \mathrm{H}_{29} \mathrm{NO}_{2}(\mathrm{M}+\mathrm{H})^{+}$ 388.22, found 388.39.

\section{Ethyl 2-(dibenzylamino)-3-methylbutanoate (5f)}

colorless oil; ${ }^{1} \mathrm{HNMR}\left(\mathrm{CDCl}_{3}\right) \delta 0.78$ (d, J=6.3 Hz, 3H), 1.01 (d, J=6.6 Hz, 3H), 1.36 (t, $J=14.2 \mathrm{~Hz}, 3 \mathrm{H}$ ), 2.10-2.21 (m, 1H), 2.83 (d, $J=13.9 \mathrm{~Hz}, 1 \mathrm{H}$ ), 3.29 (d, $J=10.9 \mathrm{~Hz}, 2 \mathrm{H}$ ), 3.97 (d, $J=13.9 \mathrm{~Hz}, 2 \mathrm{H}), 4.15-4.34$ (m, 2H), 7.20-7.40 (m, 10H); ${ }^{13} \mathrm{CNMR}\left(\mathrm{CDCl}_{3}\right) \delta \quad 14.7,19.5,19.9$, 27.2, 54.6, 59.7, 68.1, 126.9, 128.2, 128.8, 139.6, 171.9; IR (neat) 2964, 1720, 1451, 1371, 1145, 1026, 699, $451 \mathrm{~cm}^{-1}$; HRMS (ESI): Calculated for $\mathrm{C}_{21} \mathrm{H}_{27} \mathrm{NO}_{2}(\mathrm{M}+\mathrm{H})^{+} 326.20$, found 326.26.

\section{Ethyl 2-cyclohexyl-2-(dibenzylamino)acetate (5g)}

colorless oil; ${ }^{1} \mathrm{HNMR}\left(\mathrm{CDCl}_{3}\right) \delta \quad 0.78-0.82$ (m, 2H), 1.07-1.25 (m, 4H), 1.35 (t, J=14.2 Hz, 3H), 1.46-1.68 (m, 3H), 1.87-1.89 (m, 1H), 2.26 (d, $J=13.8 \mathrm{~Hz}, 1 \mathrm{H}), 2.99$ (dd, $J=17.1 \mathrm{~Hz}, J=6.1$ Hz, 1H), 3.34 (d, J=14.0 Hz, 2H), 3.97 (d, J=14.0 Hz, 2H), 4.14-4.32 (m, 2H), 7.17-7.39 (m, $10 \mathrm{H}) ;{ }^{13} \mathrm{CNMR}\left(\mathrm{CDCl}_{3}\right) \delta \quad 14.8,25.8,25.9,26.5,29.7,30.4,36.4,54.6,59.6,66.9,126.9$, 128.2, 128.8, 139.7, 171.9; IR (neat) 2930, 2853, 1718, 1264, 1143, 909, 775, $436 \mathrm{~cm}^{-1}$; HRMS (ESI): Calculated for $\mathrm{C}_{24} \mathrm{H}_{31} \mathrm{NO}_{2}(\mathrm{M}+\mathrm{H})^{+} 366.24$, found 366.35.

\section{Ethyl 2-(dibenzylamino)-2-phenylacetate (5i)}

colorless oil; ${ }^{1} \mathrm{HNMR}\left(\mathrm{CDCl}_{3}\right) \delta 1.30$ (t, $\left.J=14.2 \mathrm{~Hz}, 3 \mathrm{H}\right), 3.73$ (d, $\left.J=14.2 \mathrm{~Hz}, 2 \mathrm{H}\right), 3.75$ (d, $J=14.4 \mathrm{~Hz}, 2 \mathrm{H}), 4.20-4.34$ (m, 2H), 4.60 (s, 1H), 7.19-7.35 (m, 15H); ${ }^{13} \mathrm{CNMR}\left(\mathrm{CDCl}_{3}\right) \delta$ 14.4, 54.2, 60.4, 65.8, 127.0,127.7, 128.2, 128.3, 128.8, 136.8, 139.6, 172.1; IR (neat) 3030, 1728, 1451, 1214, 1133, 1026, 761, 669, $480 \mathrm{~cm}^{-1}$; HRMS (ESI): Calculated for $\mathrm{C}_{24} \mathrm{H}_{25} \mathrm{NO}_{2}$ 
$(\mathrm{M}+\mathrm{H})^{+}$360.19, found 360.54.

\section{Ethyl 2-(dibenzylamino)-2-p-tolylacetate (5j)}

colorless oil; ${ }^{1} \mathrm{HNMR}\left(\mathrm{CDCl}_{3}\right) \delta 1.29$ (t, $\left.J=14.2 \mathrm{~Hz}, 3 \mathrm{H}\right), 2.32$ (s, 3H), 3.74 (d, $J=14.4 \mathrm{~Hz}$, 2H), 3.76 (d, $J=14.5 \mathrm{~Hz}, 2 \mathrm{H}), 4.18-4.32$ (m, 2H), 4.56 (s, 1H), 7.12-7.36 (m, 15H); ${ }^{13} \mathrm{CNMR}$ $\left(\mathrm{CDCl}_{3}\right) \delta$ 14.7, 21.4, 54.4, 60.0, 65.8, 127.2, 128.5, 129.0, 129.1, 129.4, 133.6,137.7, 140.0, 172.6; IR (neat) 3030, 1727, 1452, 1214, 1132, 762, $449 \mathrm{~cm}^{-1}$; HRMS (ESI): Calculated for $\mathrm{C}_{25} \mathrm{H}_{27} \mathrm{NO}_{2}(\mathrm{M}+\mathrm{H})^{+}$374.20, found 374.34.

\section{Ethyl 2-(4-chlorophenyl)-2-(dibenzylamino)acetate (5k)}

colorless oil; ${ }^{1} \mathrm{HNMR}\left(\mathrm{CDCl}_{3}\right) \delta 1.31$ (t, $\left.J=13.9 \mathrm{~Hz}, 3 \mathrm{H}\right), 3.74$ (d, $\left.J=14.0 \mathrm{~Hz}, 2 \mathrm{H}\right), 3.75$ (d, $J=14.0 \mathrm{~Hz}, 2 \mathrm{H}), 4.20-4.32$ (m, 2H), 4.54 (s, 1H), 7.23-7.33 (m, 14H); ${ }^{13} \mathrm{CNMR}\left(\mathrm{CDCl}_{3}\right) \delta$ 14.4, 54.2, 60.6, 65.2, 127.1, 128.3, 128.5, 128.8, 130.1, 133.6, 135.4, 139.3, 171.6; IR (neat) 3032, 2985, 2847, 1727, 1599, 1491, 1451, 1371, 1264, 1176, 1132, 1094, 1021, 909, 701, 673, 666, 460, 426, $409 \mathrm{~cm}^{-1}$; HRMS (ESI): Calculated for $\mathrm{C}_{24} \mathrm{H}_{24} \mathrm{ClNO}_{2}(\mathrm{M}+\mathrm{H})^{+}$394.15, found 395.16.

\section{Ethyl 2-(dibenzylamino)-2-(4-methoxyphenyl)acetate (5l)}

colorless oil; ${ }^{1} \mathrm{HNMR}\left(\mathrm{CDCl}_{3}\right) \delta 1.29$ (t, J=14.7 Hz, 3H), 3.74 (d, J=14.0 Hz, 2H), 3.75 (d, $J=14.0 \mathrm{~Hz}, 2 \mathrm{H}$ ), 3.79 (s, 3H), 4.20-4.32 (m, 2H), 4.54 (s, 1H), 6.86 (dd, J=9.2 Hz, J=2.4 Hz, 2H), 7.20-7.46 (m, 12H); ${ }^{13} \mathrm{CNMR}\left(\mathrm{CDCl}_{3}\right) \delta$ 14.4, 54.1, 55.2, 60.3, 65.2, 113.7, 126.9, 128.2, 128.8, 130.0, 139.7, 159.1, 172.3; IR (neat) 3032, 2984, 2848, 1724, 1599, 1491, 1451, 1371, 1267, 1165, 1132, 1094, 1021, 909, 701, 673, 666, 460, 426, $409 \mathrm{~cm}^{-1}$; HRMS (ESI): Calculated for $\mathrm{C}_{25} \mathrm{H}_{27} \mathrm{NO}_{3}(\mathrm{M}+\mathrm{H})^{+}$390.20, found 390.37.

\section{Ethyl 2-cyclopropyl-2-(dibenzylamino)acetate (5m)}

colorless oil; ${ }^{1} \mathrm{HNMR}\left(\mathrm{CDCl}_{3}\right) \delta$ 0.17-0.26 (m, 2H), 0.52-0.61 (m, 2H), 1.23-1.28 (m, 1H), 1.33 (t, $J=14.0 \mathrm{~Hz}, 3 \mathrm{H}), 2.48$ (d, $J=9.8 \mathrm{~Hz}, 1 \mathrm{H}$ ), 3.62 (d, $J=14.0 \mathrm{~Hz}, 2 \mathrm{H}), 4.04$ (d, $J=14.0 \mathrm{~Hz}$, 2H), 4.16-4.29 (m, 2H), 7.20-7.39 (m, 10H); ${ }^{13} \mathrm{CNMR}\left(\mathrm{CDCl}_{3}\right) \delta \quad 4.0,4.2,11.3,14.6,54.8$, 60.0, 66.3, 126.9, 128.1, 128.7, 140.0, 172.7; IR (neat) 3019, 2933, 2843, 1721, 1493, 1370, 1216, 1154, 1101, 1073, 1026, 971, 909, 731, 700, 669, 469, $441 \mathrm{~cm}^{-1}$; HRMS (ESI): Calculated for $\mathrm{C}_{21} \mathrm{H}_{25} \mathrm{NO}_{2}(\mathrm{M}+\mathrm{H})^{+}$324.19, found 324.28.

\section{Ethyl 2-(dibenzylamino)-3-(trimethylsilyl)propanoate (5n)}

colorless oil; ${ }^{1} \mathrm{HNMR}\left(\mathrm{CDCl}_{3}\right) \delta 0.00$ (s, 9H), 0.97 (dd, $J=14.7 \mathrm{~Hz}, J=3.7 \mathrm{~Hz}, 1 \mathrm{H}$ ), 1.42 (dd, $J=14.0 \mathrm{~Hz}, J=11.0 \mathrm{~Hz}, 1 \mathrm{H}), 1.47$ (t, $J=14.7 \mathrm{~Hz}, 3 \mathrm{H}$ ), 3.56 (dd, $J=11.0 \mathrm{~Hz}, J=3.7 \mathrm{~Hz}, 1 \mathrm{H}$ ), 3.61 (d, $J=13.0 \mathrm{~Hz}, 2 \mathrm{H}), 4.04$ (d, $J=13.4 \mathrm{~Hz}, 2 \mathrm{H}), 4.25-4.42$ (m, 2H), 7.32-7.51 (m, 10H); ${ }^{13} \mathrm{CNMR}$ $\left(\mathrm{CDCl}_{3}\right) \delta \quad 0.00,16.0,19.0,55.6,59.0,61.5,128.1,128.3,129.2,129.6,130.2,141,9,174.5$; 
IR (neat) 3019, 1722, 1493, 1452, 1370, 1249, 1215, 1149, 1103, 1062, 854, 756, 670, 507 $\mathrm{cm}^{-1}$; HRMS (ESI): Calculated for $\mathrm{C}_{22} \mathrm{H}_{31} \mathrm{NO}_{2} \mathrm{Si}(\mathrm{M}+\mathrm{H})^{+} 370.21$, found 370.15 .

\section{Ethyl 2-(dibenzylamino)hex-5-enoate (5o)}

colorless oil; ${ }^{1} \mathrm{HNMR}\left(\mathrm{CDCl}_{3}\right) \delta 1.34$ (t, J=7.26 Hz, 3H), 1.55-1.85 (m, 2H), 1.90-2.04 (m, 1H), 2.14-2.24 (m, 1H), 3.32 (t, $J=7.26 \mathrm{~Hz}, 1 \mathrm{H}), 3.57$ (d, $J=13.86 \mathrm{~Hz}, 2 \mathrm{H}$ ), 3.90 (d, $J=13.86 \mathrm{~Hz}$, 2H), 4.84-4.92 (m, 2H), 5.59-5.74 (m, 1H), 7.20-7.38 (m, 10H); ${ }^{13} \mathrm{CNMR}\left(\mathrm{CDCl}_{3}\right) \delta$ 14.6, 28.9, 30.3, 54.6, 60.0, 60.3, 114.9, 126.9, 128.2, 128.9, 137.9, 139.7, 172.1; IR (neat) 3019, 1721, 1494, 1453, 1373, 1219, 1181, 1145, 1027, 917, 783, 769, 752, 736, 728, 699, 671, 449, $424 \mathrm{~cm}^{-1}$; HRMS (ESI): Calculated for $\mathrm{C}_{22} \mathrm{H}_{27} \mathrm{NO}_{2}(\mathrm{M}+\mathrm{H})^{+} 338.20$, found 338.21.

\section{Ethyl 8-(4-methoxyphenoxy)-2-(dibenzylamino)octanoate (5p)}

colorless oil; ${ }^{1} \mathrm{HNMR}\left(\mathrm{CDCl}_{3}\right) \delta$ 1.18-1.45 (m, 9H), 1.65-1.75 (m, 4H), 3.29 (t, J=14.7 Hz, 1H), 3.54 (d, $J=14.0 \mathrm{~Hz}, 2 \mathrm{H}), 3.81$ (s, 3H), 3.86 (t, $J=13.4 \mathrm{~Hz}, 2 \mathrm{H}), 3.91$ (d, $J=14.0 \mathrm{~Hz}, 2 \mathrm{H}$ ), 4.12-4.29 (m, 2H), 6.82 (s, 4H), 7.21-7.37 (m, 10H); ${ }^{13} \mathrm{CNMR}\left(\mathrm{CDCl}_{3}\right) \delta$ 14.6, 25.9, 26.0, 29.0, 29.3, 29.4, 54.5, 55.7, 60.0, 60.5, 68.5, 114.6, 115.4, 126.9, 128.2, 128.8, 139.8, 153.2, 173.1; IR (neat) 3033, 2935, 1720, 1507, 1218, 782, 763, 734, 676, 669, 662, 494, 465, 451 $\mathrm{cm}^{-1}$; HRMS (ESI): Calculated for $\mathrm{C}_{31} \mathrm{H}_{39} \mathrm{NO}_{3}(\mathrm{M}+\mathrm{H})^{+} 490.29$, found 490.42 .

\section{Ethyl 2-(dibenzylamino)-4-methylpent-4-enoate (6a)}

Typical procedure (Table 3, entry 2): Under an argon atmosphere, a mixture of 1-ethoxy-2-dibenzylamino-1-tert-butyldimethylsiloxyethylene $(59.6 \mathrm{mg}, 0.15 \mathrm{mmol})$ and tetramethallyltin $(25.4 \mathrm{mg}, 0.075 \mathrm{mmol})$ in DME $(2.0 \mathrm{ml})$ and diethylaluminium chloride $(0.31$ $\mathrm{ml}, 0.30 \mathrm{mmol}, 0.98 \mathrm{M}$ in hexane ) were added to a solution of DDQ (35.1 $\mathrm{mg}, 0.15 \mathrm{mmol}$ ) in DME $(2.0 \mathrm{ml})$ at $-50{ }^{\circ} \mathrm{C}$. The reaction mixture was allowed to warm to ambient temperature with stirring for $18 \mathrm{~h}$. The reaction was quenched with $10 \%$ aqNa $\mathrm{SO}_{3}$, and the whole mixture was extracted with ethyl acetate $(10 \mathrm{ml} \times 3)$. The combined extracts were washed with brine, dried over anhydrous $\mathrm{Na}_{2} \mathrm{SO}_{4}$, and concentrated in vacuo. The crude product was purified by preparative TLC on silica gel (EtOAc/n-hexane $=1 / 7$ ) to give ethyl 2-(dibenzylamino)-4-methylpent-4-enoate (72\%, $36.3 \mathrm{mg}$ ).

- Ethyl 2-(dibenzylamino)-4-methylpent-4-enoate (6a): colorless oil; ${ }^{1} \mathrm{HNMR}\left(\mathrm{CDCl}_{3}\right) \delta$ 1.34 (t, $J=14.0 \mathrm{~Hz}, 3 \mathrm{H}$ ), 1.49 (s, 3H), 2.49 (d, J=7.3 Hz, 2H),3.50 (d, J=14.0 Hz, 2H), 3.52 (t, $J=15.3 \mathrm{~Hz}, 1 \mathrm{H}), 3.90$ (d, J=14.0 Hz, 2H), 4.17-4.28 (m, 2H), 4.75 (d, J=35.1 Hz, 2H), 7.21-7.37 (m, 10H); ${ }^{13} \mathrm{CNMR}\left(\mathrm{CDCl}_{3}\right) \delta$ 14.6, 21.7, 38.1, 54.4, 58.9, 60.1, 113.0, 126.9, 128.1, 129.0, 139.6, 141.9, 172.5; IR (neat) 3066, 3031, 3009, 2983, 2844, 2360, 1722, 1493, 1452, 1373, 1233, 1175, 1137, 1026, 895, $473 \mathrm{~cm}^{-1}$; HRMS (ESI): Calculated for $\mathrm{C}_{22} \mathrm{H}_{27} \mathrm{NO}_{2}$ 
$(\mathrm{M}+\mathrm{H})^{+}$338.20, found 338.20.

Ethyl 2-(dibenzylamino)pent-4-enoate (6b)

Typical procedure (Table 3, entry 5): Under an argon atmosphere, a mixture of 1-ethoxy-2-dibenzylamino-1-tert-butyldimethylsiloxyethylene $(59.6 \mathrm{mg}, 0.15 \mathrm{mmol})$ and allyltributyltin (49.7 mg, $0.15 \mathrm{mmol})$ in DME (2.0 ml), and diethylaluminiumchloride (0.31 $\mathrm{ml}, 0.30 \mathrm{~mol}, 0.98 \mathrm{M}$ in hexane ) were added to a solution of DDQ (35.1 $\mathrm{mg}, 0.15 \mathrm{mmol}$ ) in DME $(0.5 \mathrm{ml})$ at $-50{ }^{\circ} \mathrm{C}$. The reaction mixture was allowed to warm to ambient temperature with stirring for $18 \mathrm{~h}$. The reaction was quenched with $10 \% \mathrm{aqNa}_{2} \mathrm{SO}_{3}$, and the whole mixture was extracted with ethyl acetate $(10 \mathrm{ml} \mathrm{x} \mathrm{3)}$. The combined extracts were washed with brine, dried over anhydrous $\mathrm{Na}_{2} \mathrm{SO}_{4}$, and concentrated in vacuo. The crude product was purified by preparative TLC on silica gel (EtOAc/n-hexane $=1 / 7$ ) to give ethyl 2-(dibenzylamino)pent-4-enoate (64\%, $31.1 \mathrm{mg})$.

- Ethyl 2-(dibenzylamino)pent-4-enoate (6b): colorless oil; ${ }^{1} \mathrm{HNMR}\left(\mathrm{CDCl}_{3}\right) \delta 1.33(\mathrm{t}$, $J=7.3 \mathrm{~Hz}, 3 \mathrm{H}$ ), 2.52 (t, $J=6.9 \mathrm{~Hz}, 2 \mathrm{H}), 3.41$ (t, $J=7.9 \mathrm{~Hz}, 1 \mathrm{H}), 3.57$ (d, $J=14.0 \mathrm{~Hz}, 2 \mathrm{H}), 3.90$ (d, $J=13.9 \mathrm{~Hz}, 2 \mathrm{H}$ ), 4.15-4.29 (m, 2H), 5.01 (s. 1H), 5.07 (d, J=6.6 Hz, 1H), 5.65-5.80 (m, 1H), 7.19-7.39 (m, 10H); ${ }^{13} \mathrm{CNMR}\left(\mathrm{CDCl}_{3}\right) \delta$ 14.6, 34.1, 54.5, 60.1, 60.7, 116.8, 127.0, 128.2, 128.8, 135.1, 139.6, 172.3; IR (neat) 3064, 3029, 2980, 2936, 2843, 1728, 1452, 1183, 1141, 1026, 914, 747, $699 \mathrm{~cm}^{-1}$; HRMS (ESI): Calculated for $\mathrm{C}_{21} \mathrm{H}_{25} \mathrm{NO}_{2}(\mathrm{M}+\mathrm{H})^{+}$324.19, found $324.43(\mathrm{M}+\mathrm{H})^{+}$.

\section{${ }^{1} \mathrm{H}$ and ${ }^{13} \mathrm{C}$ spectra}




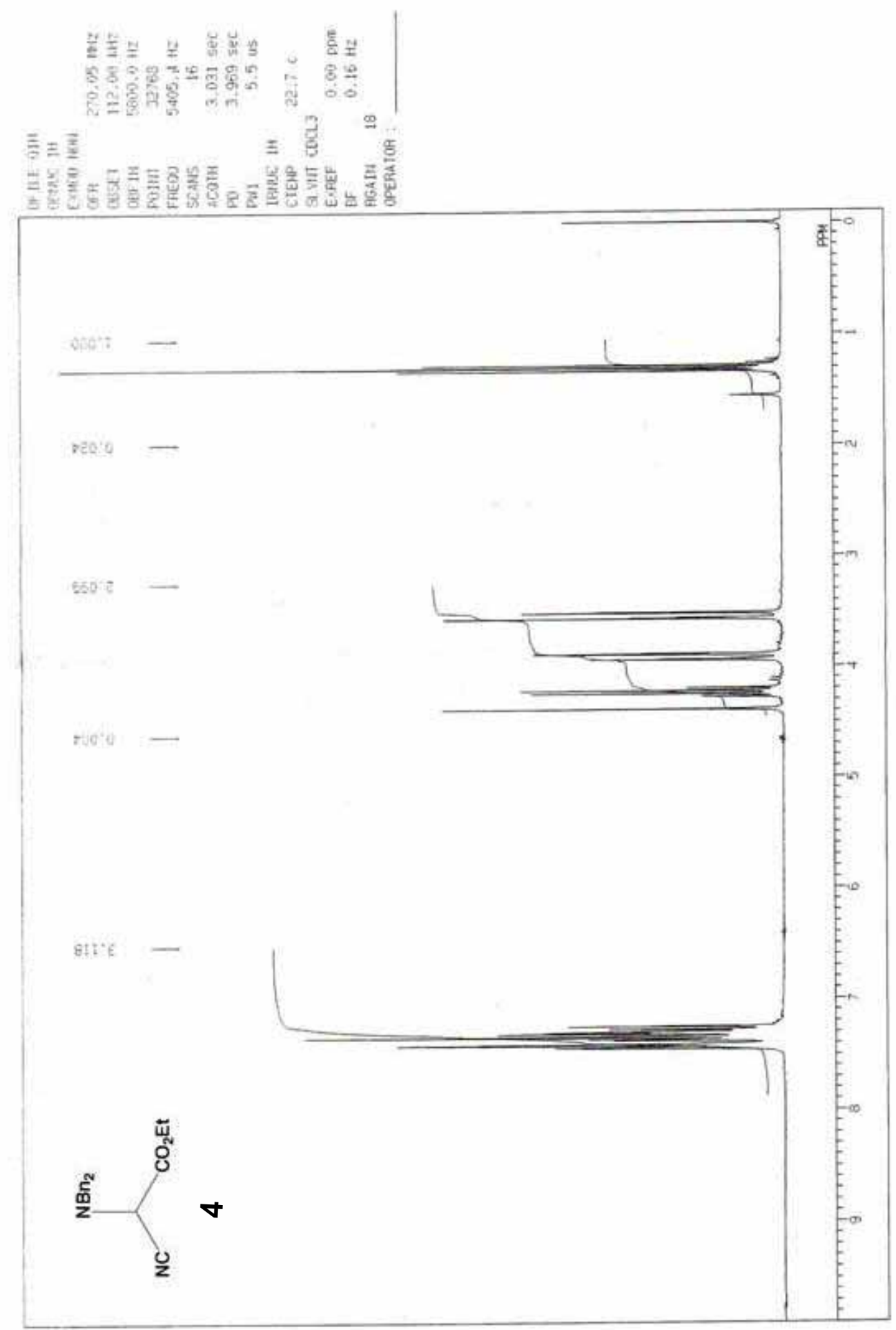




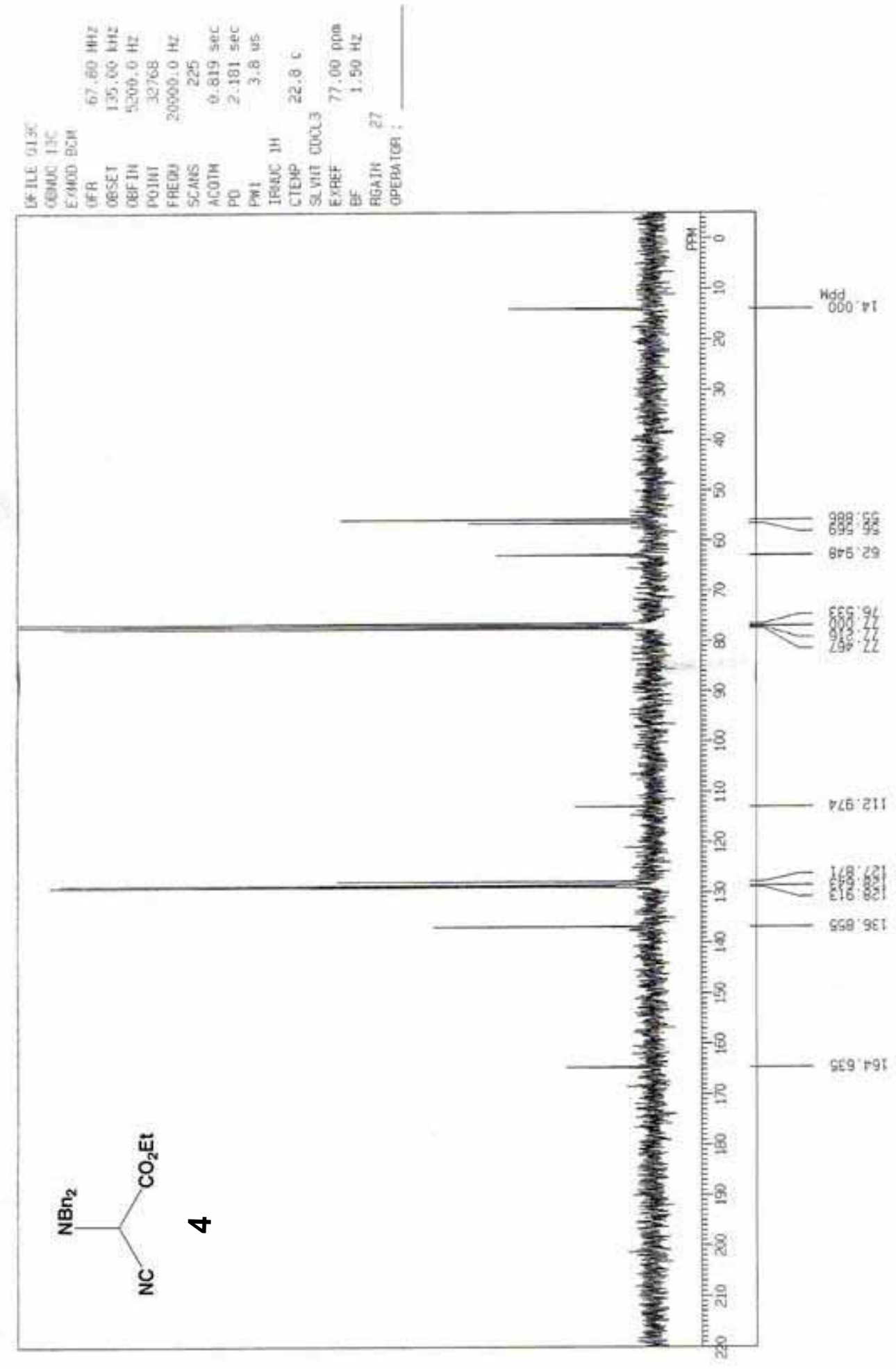




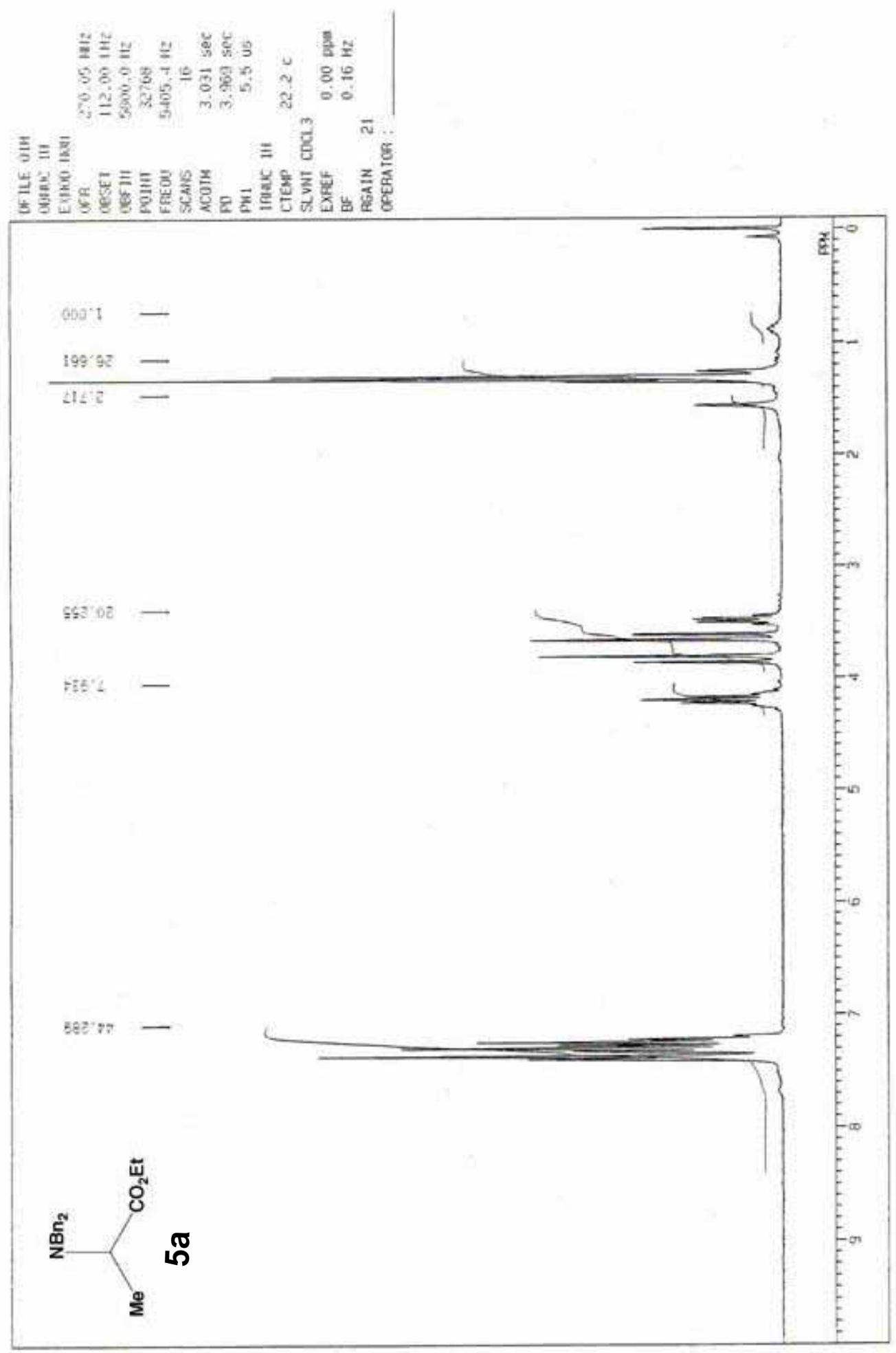




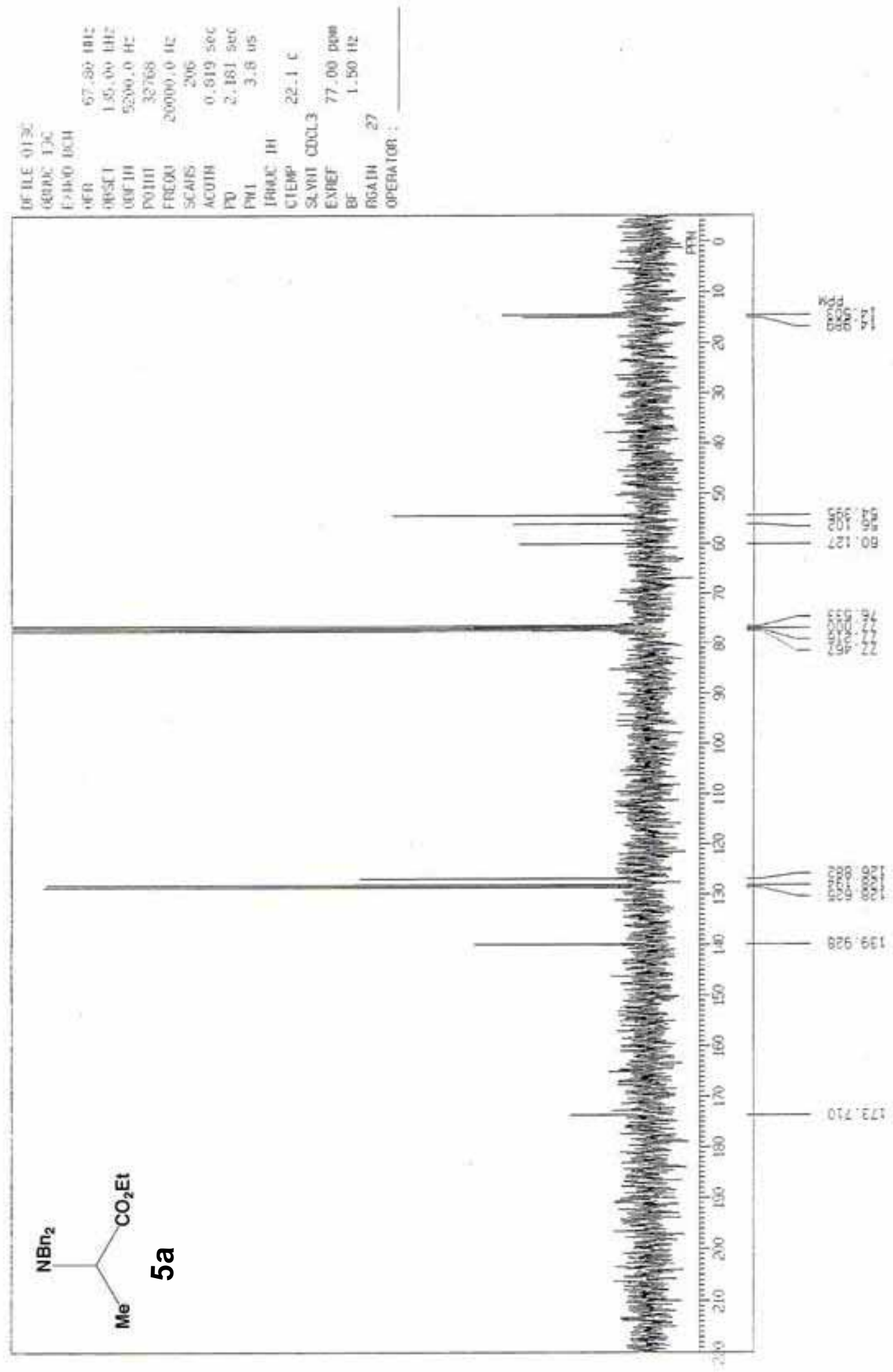




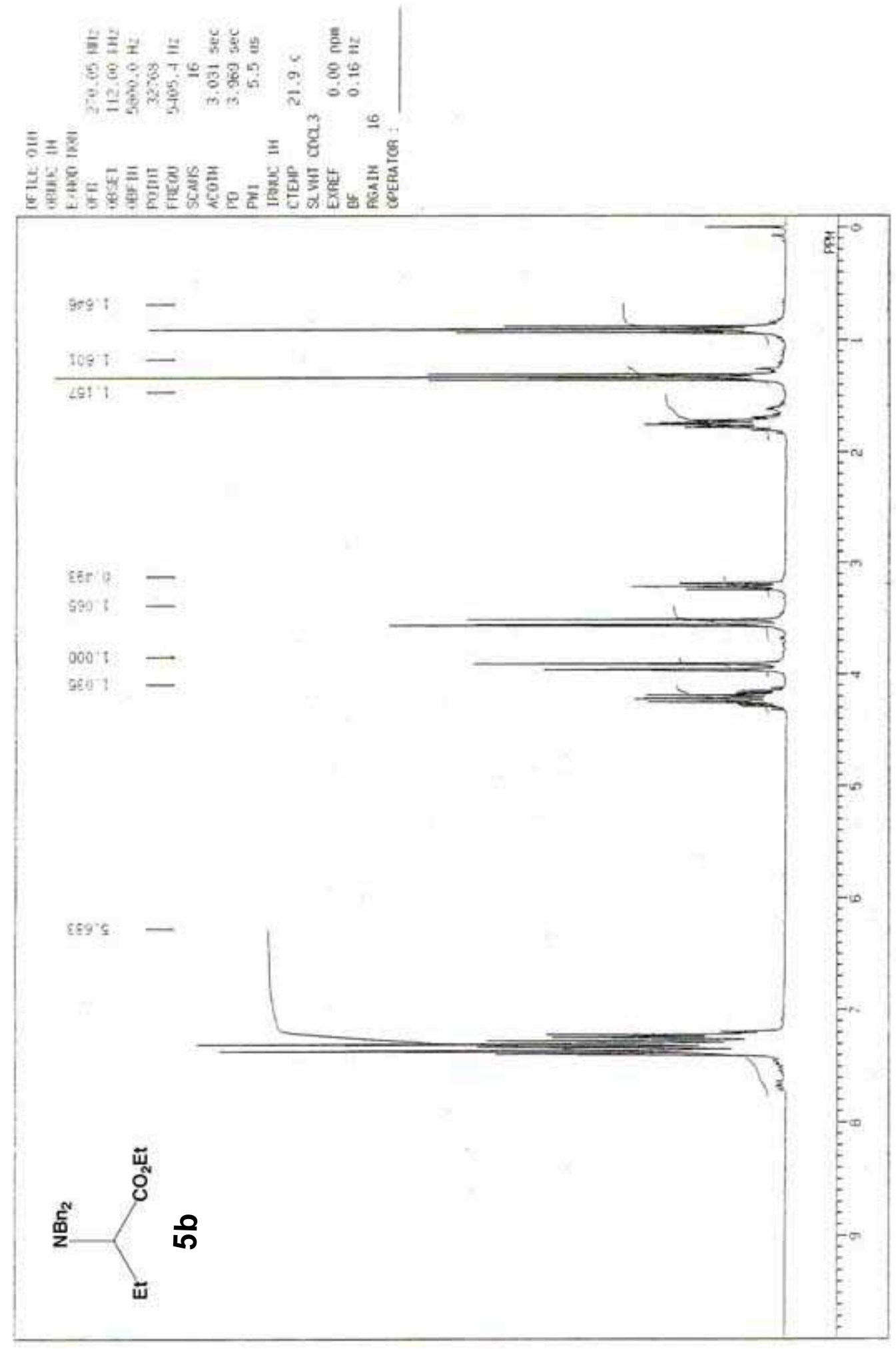




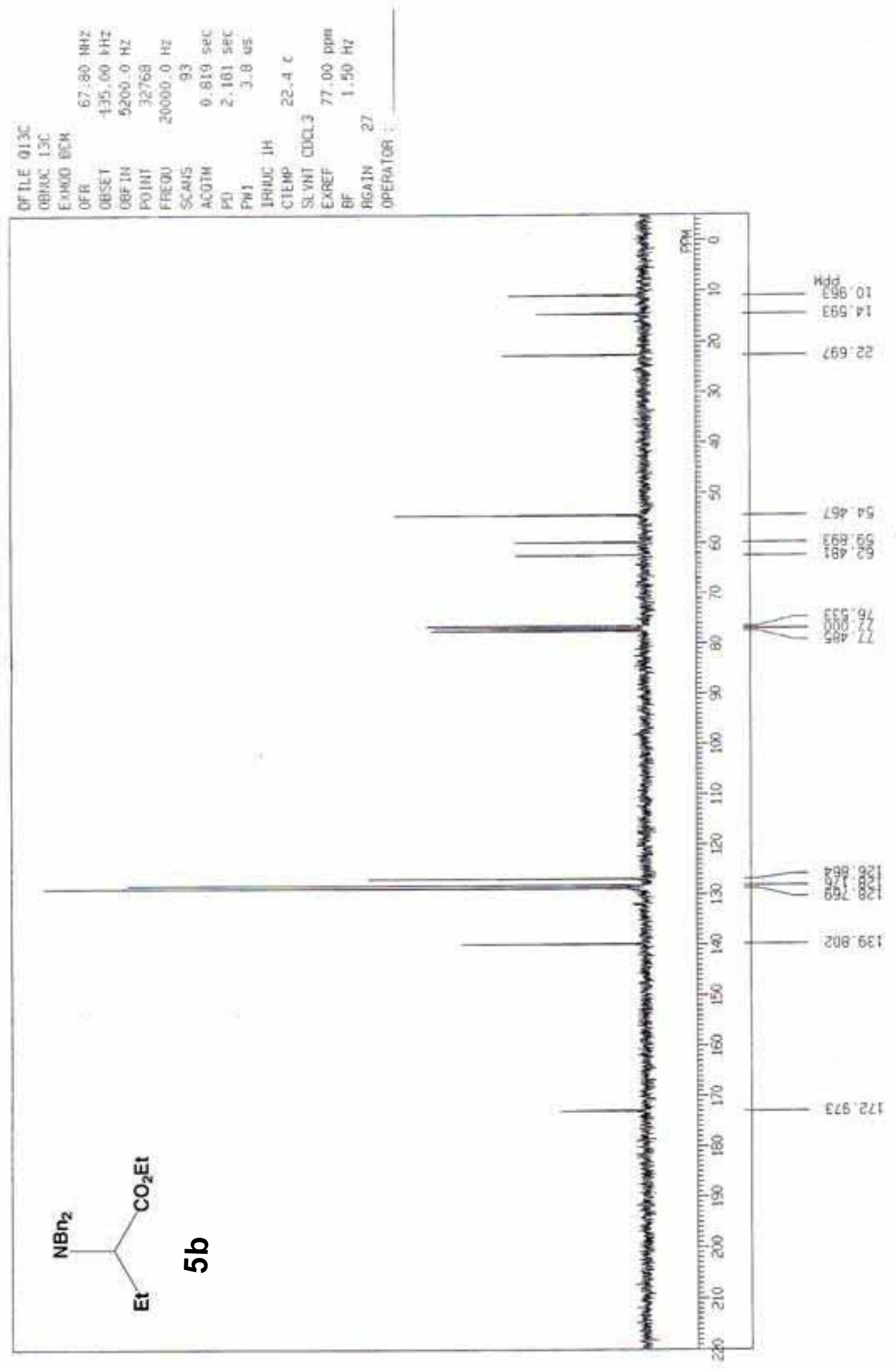




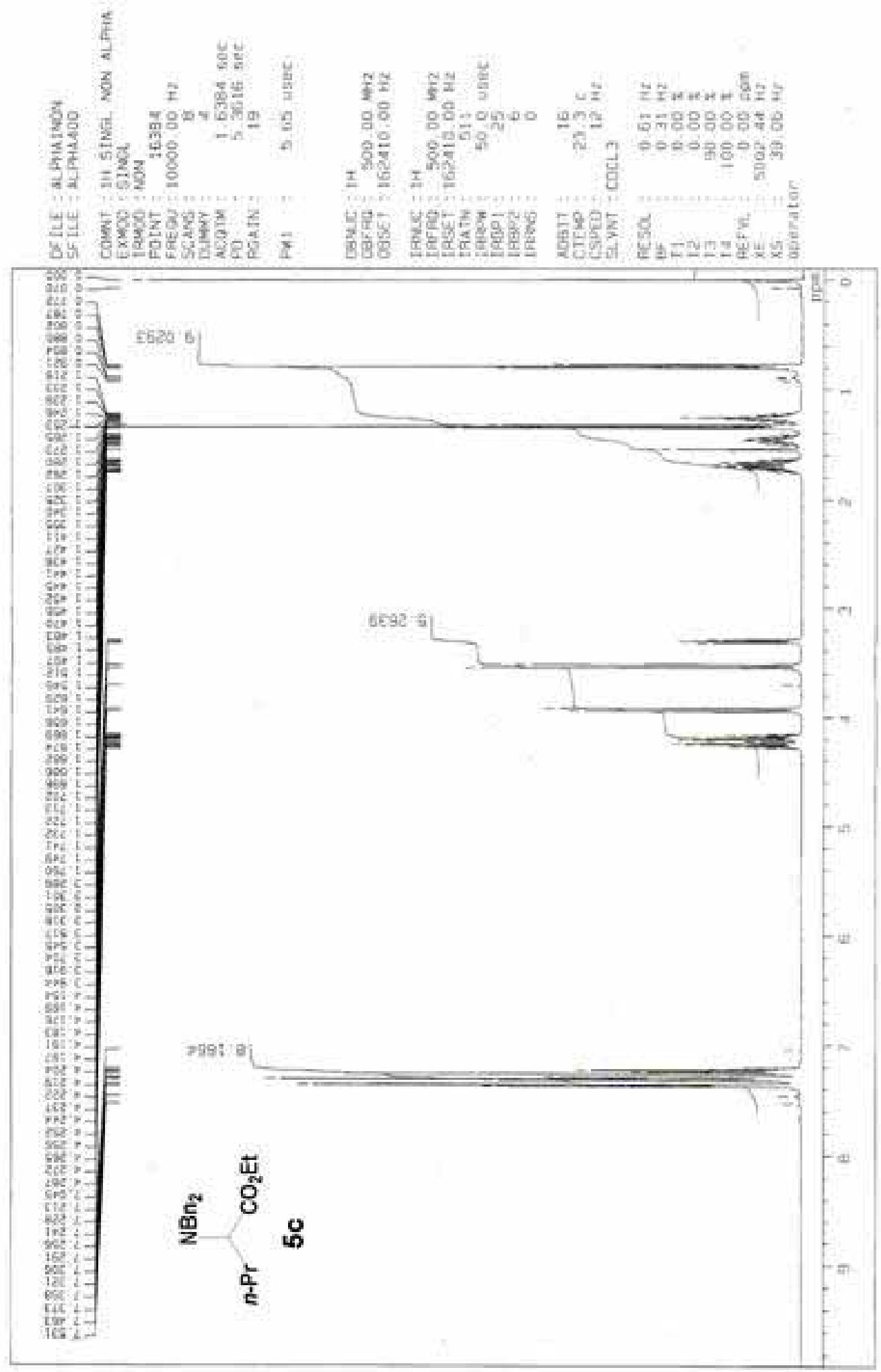




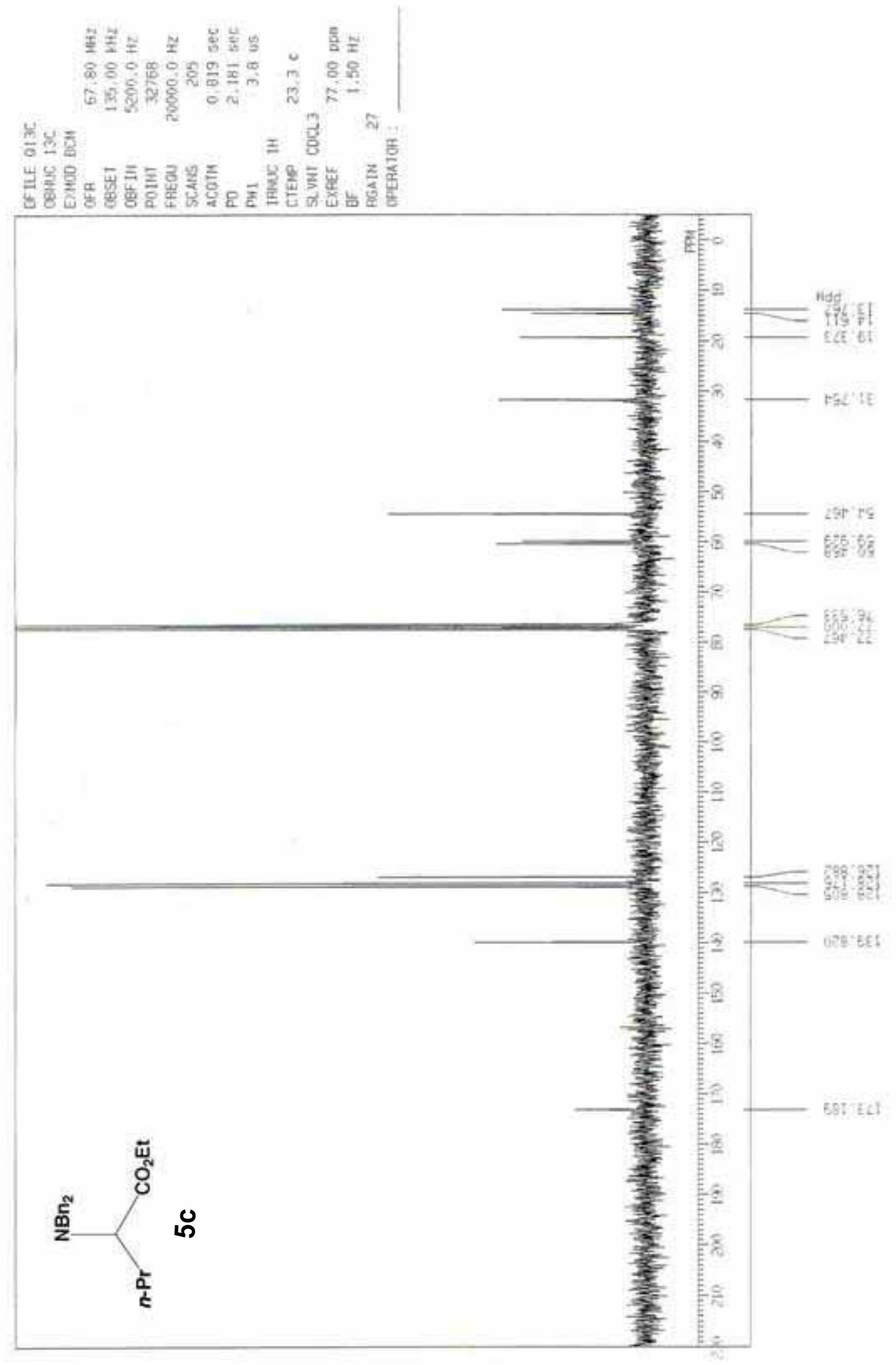




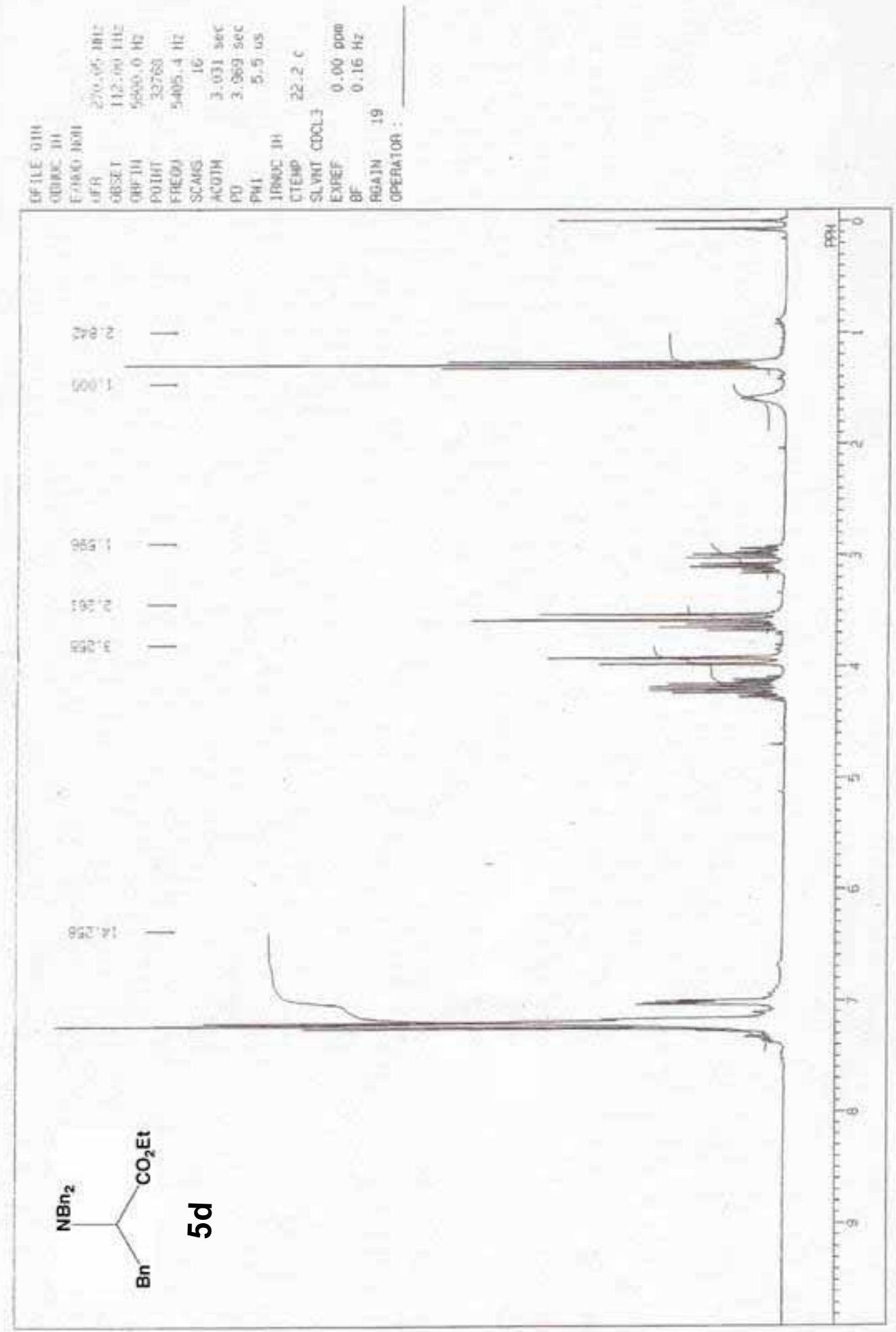




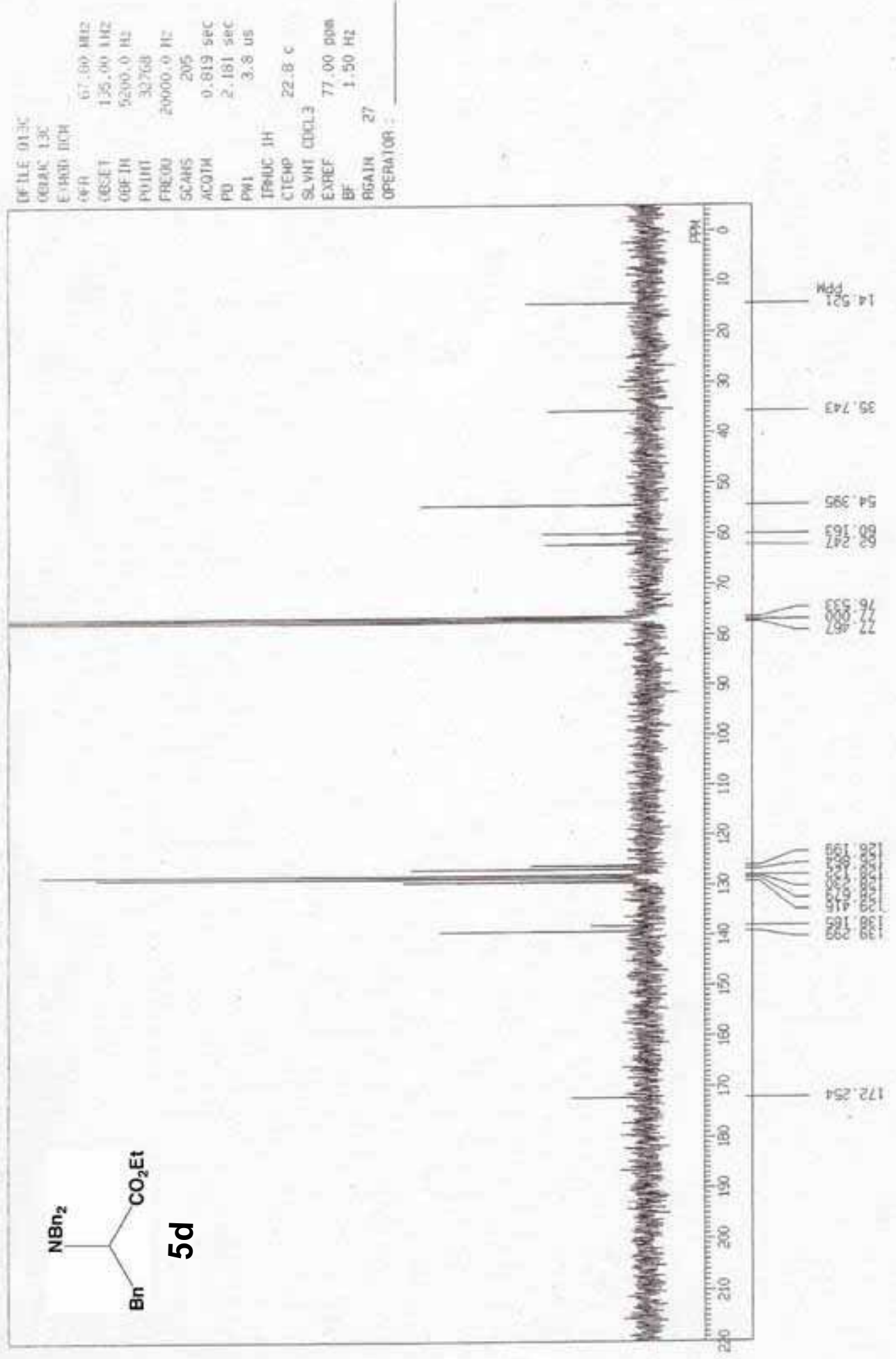




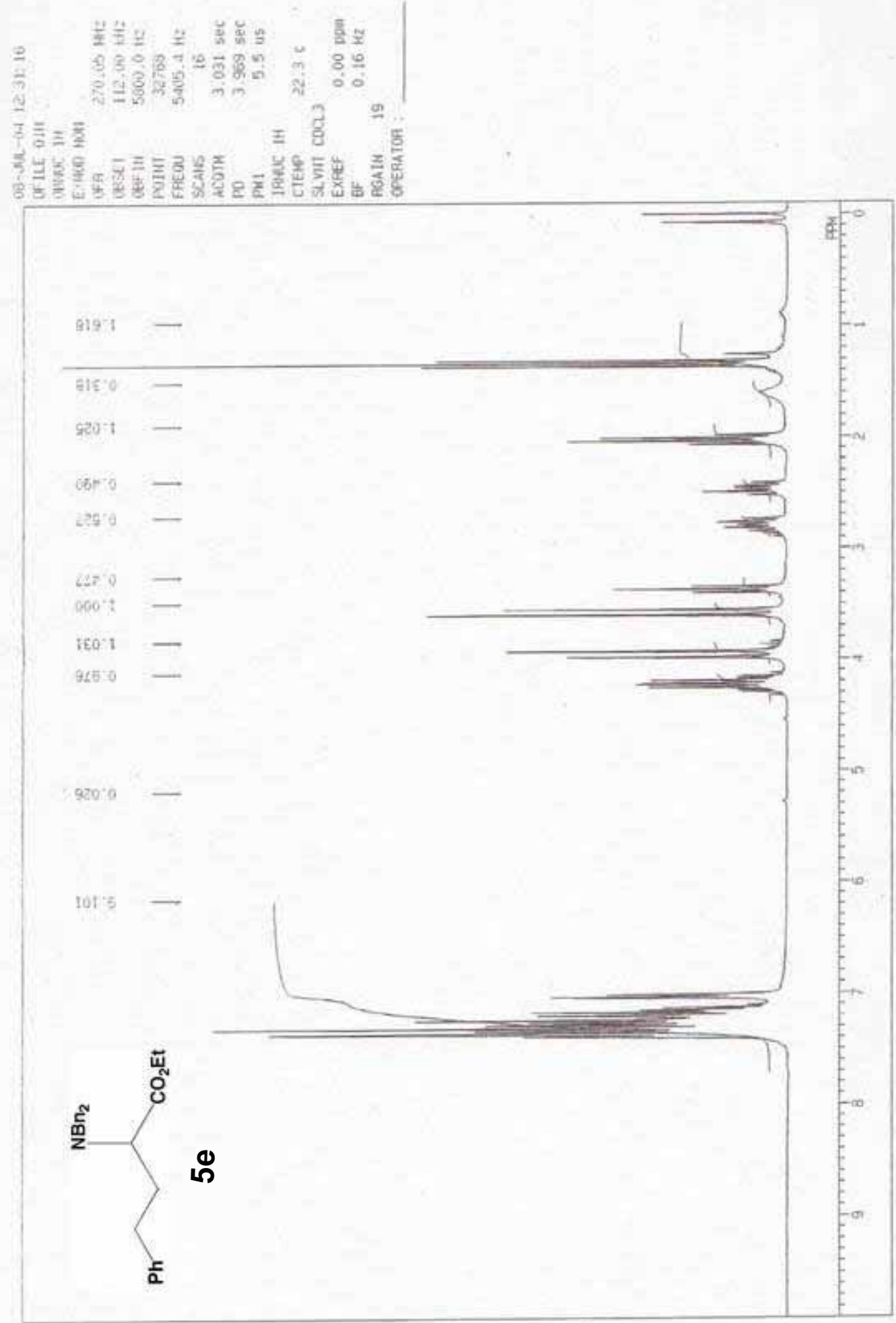




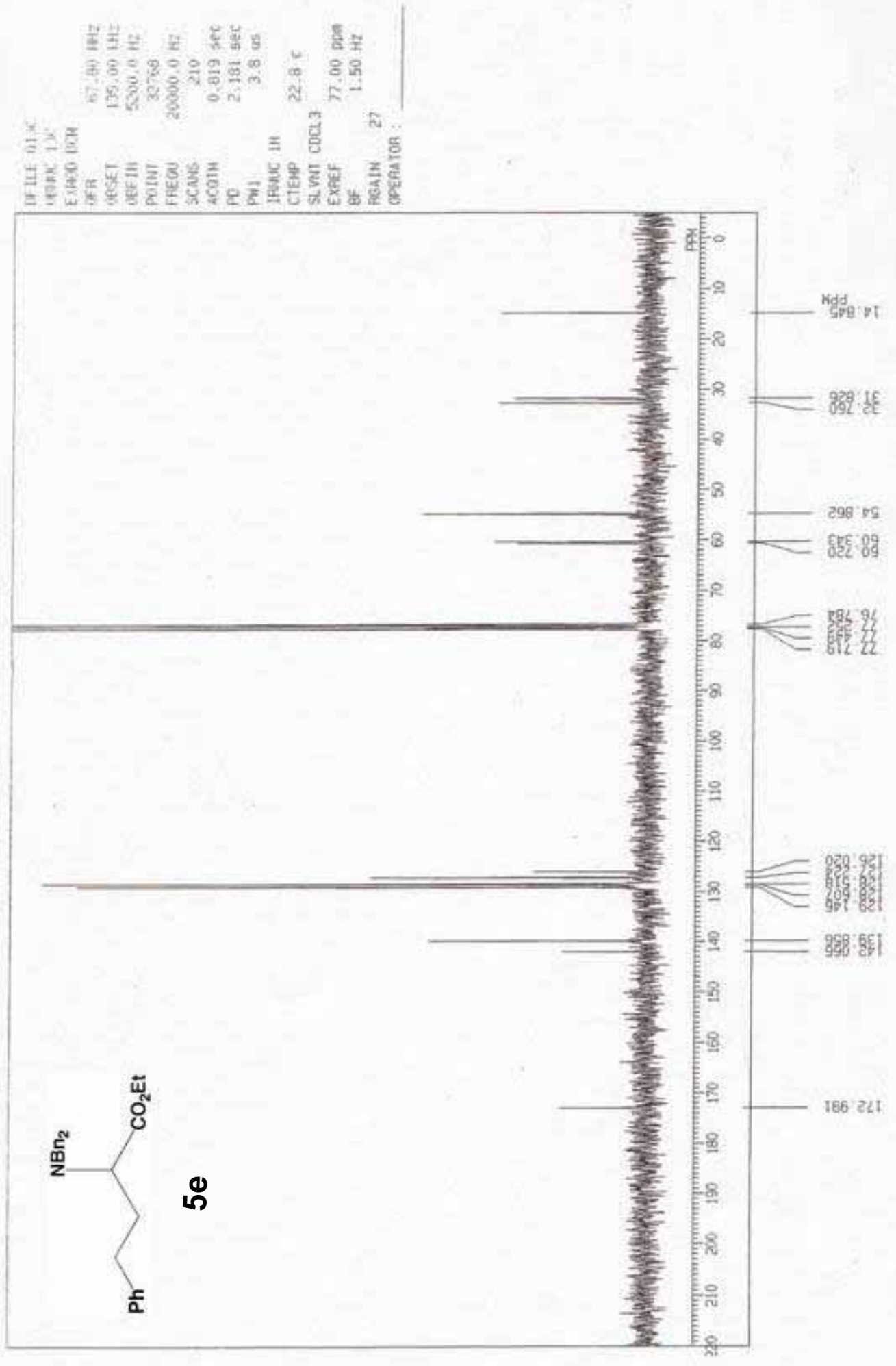




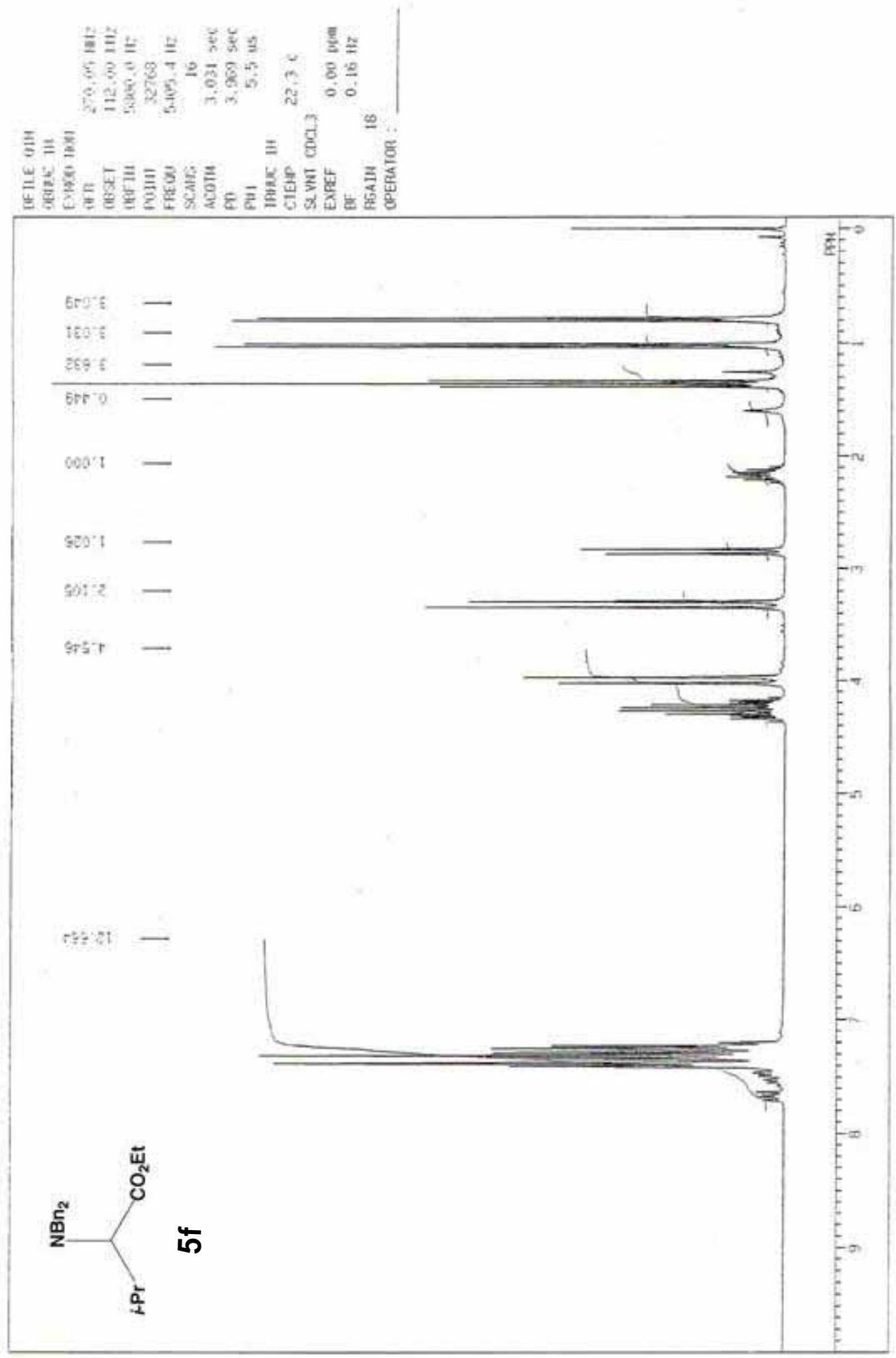


불중

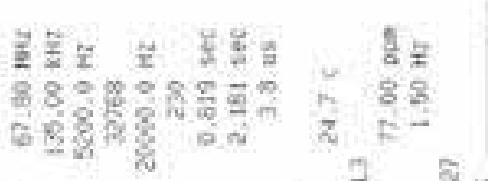

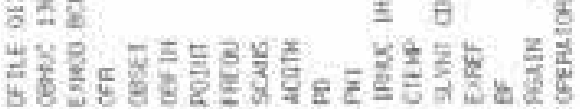

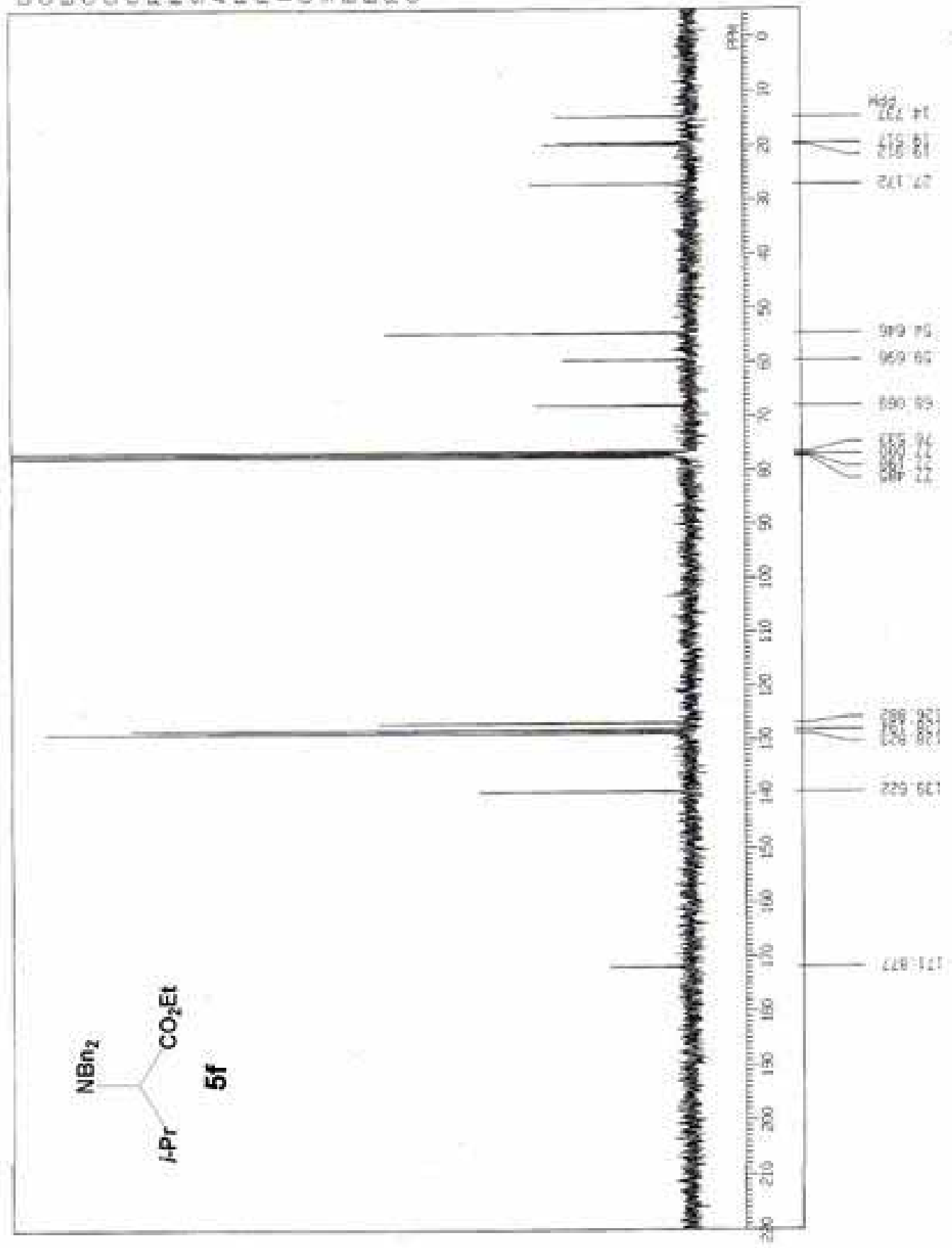




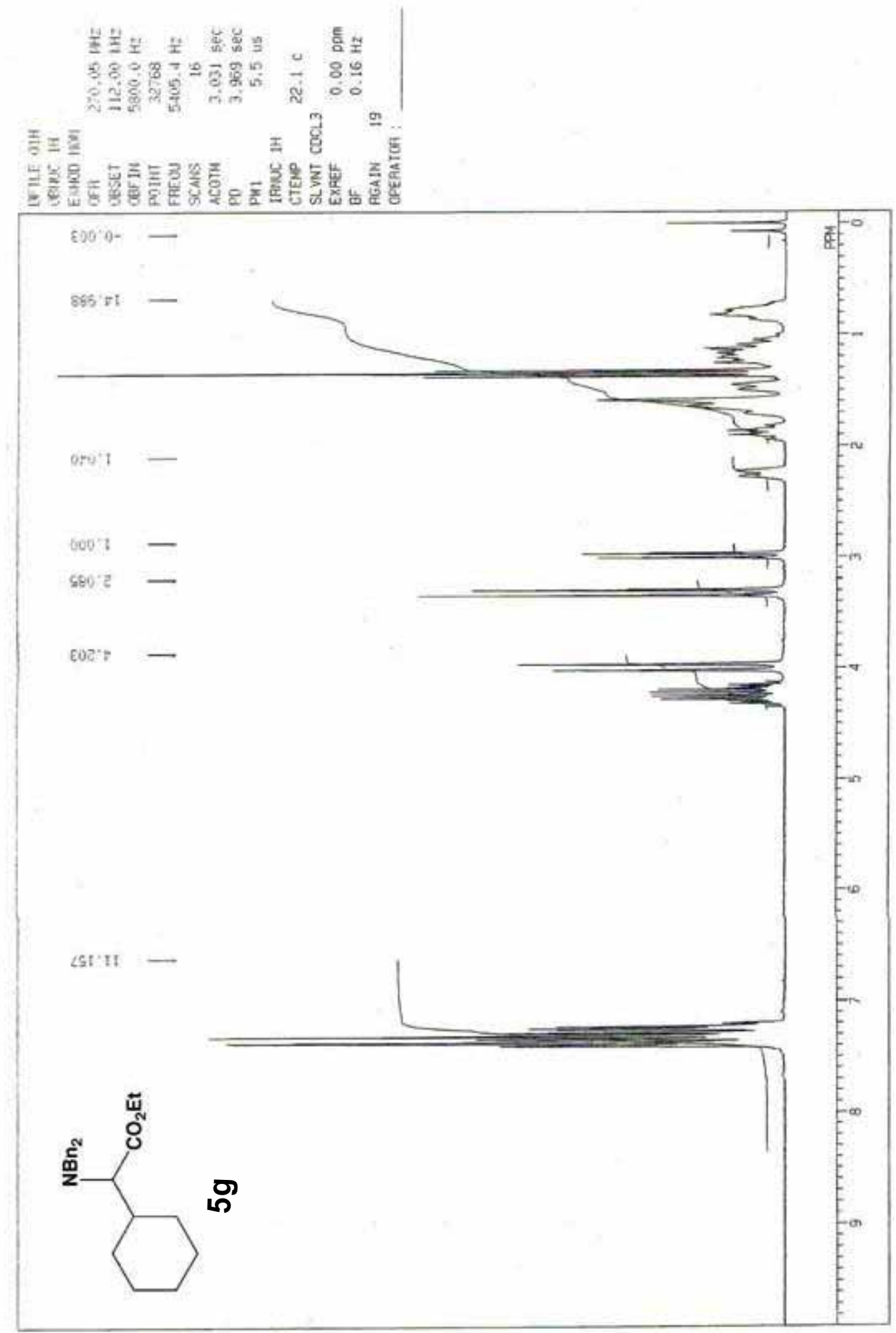




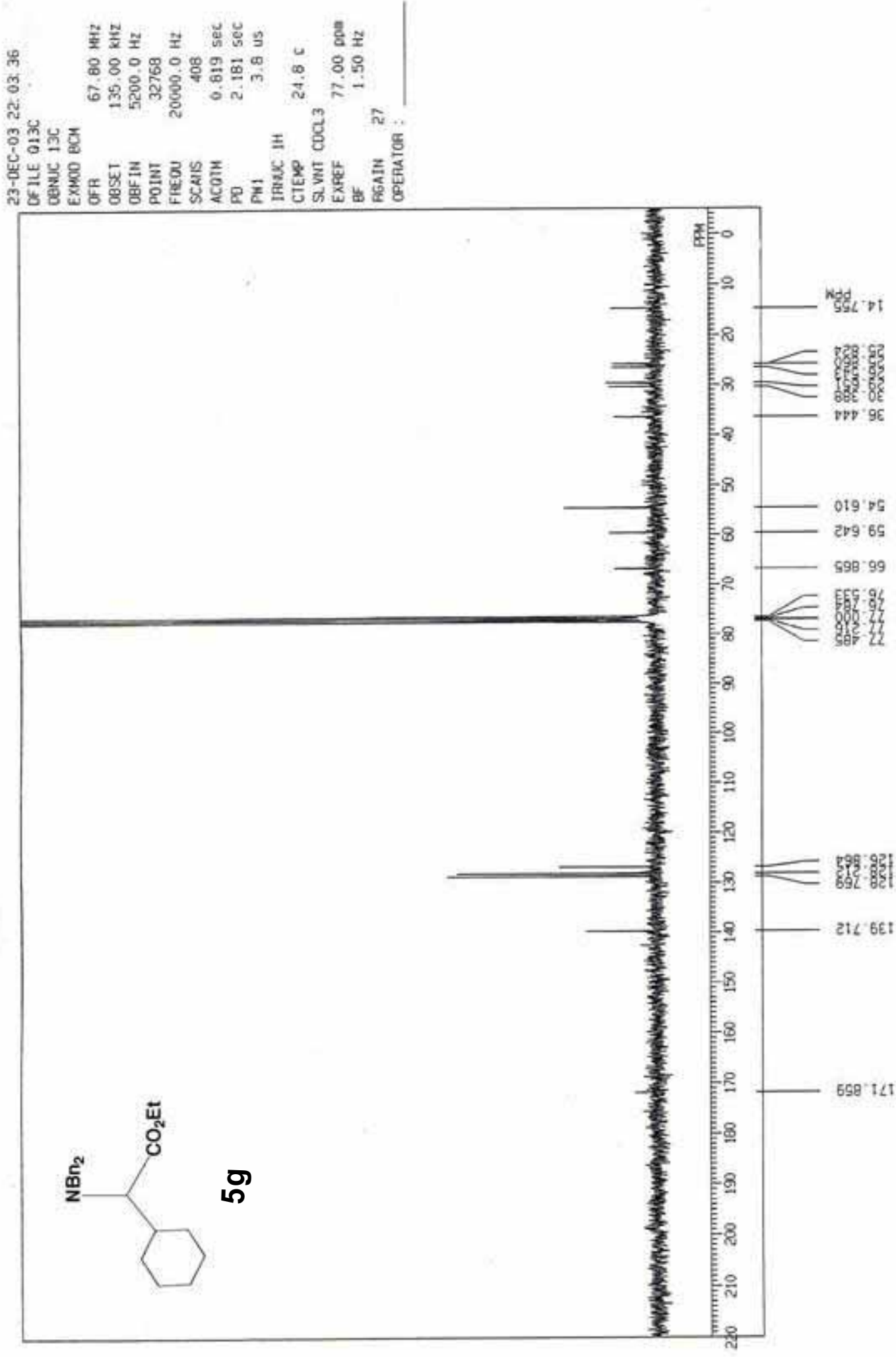




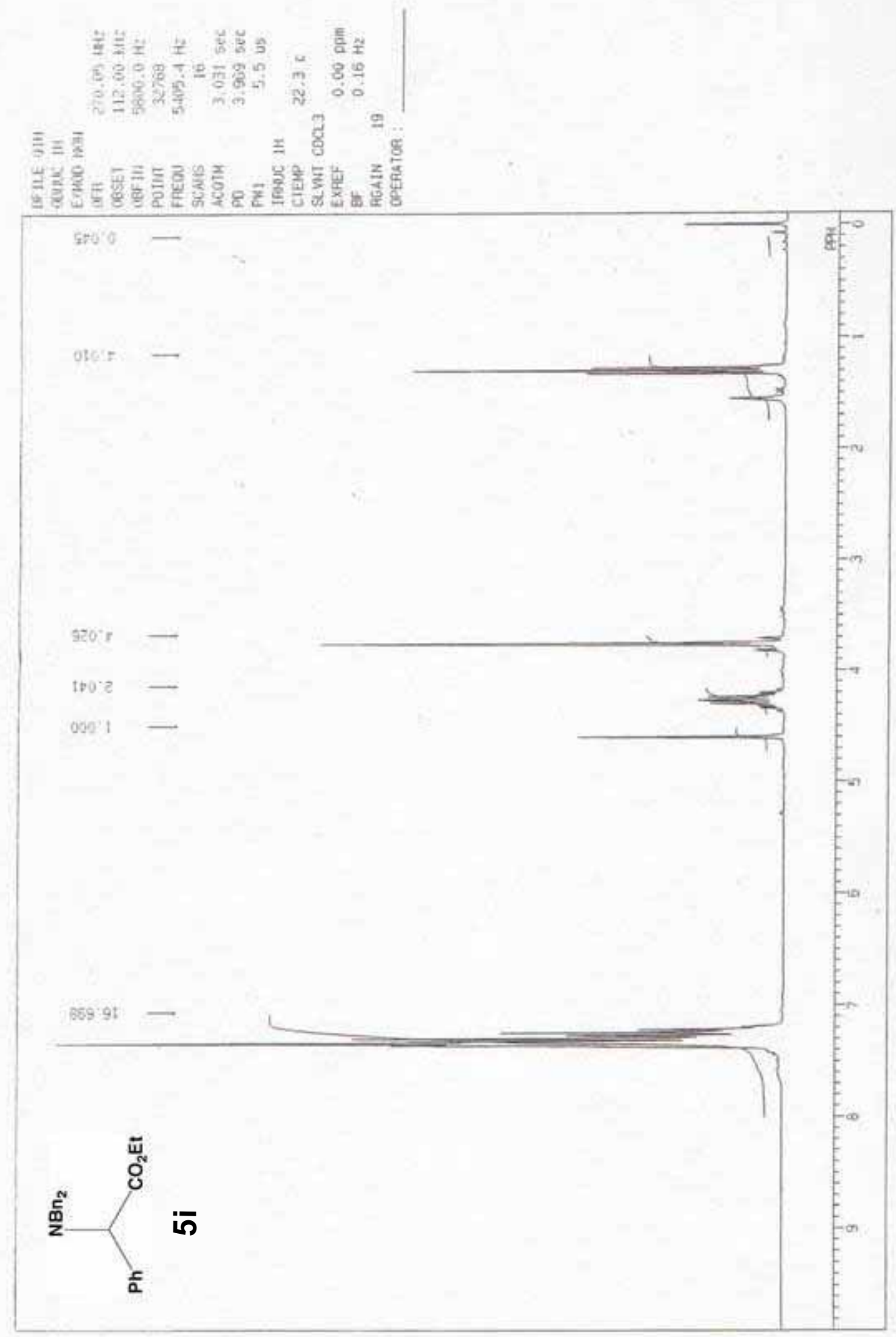




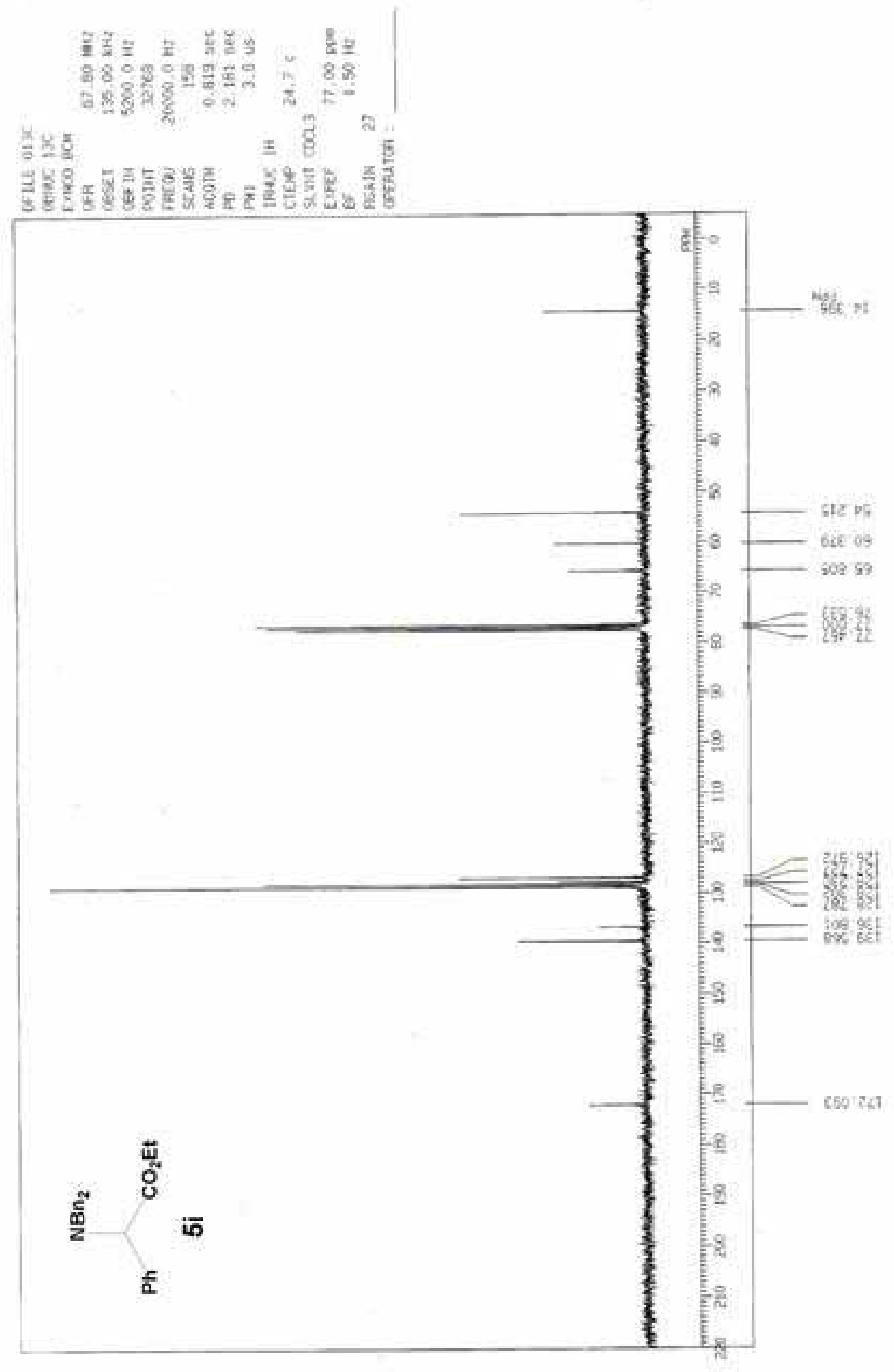




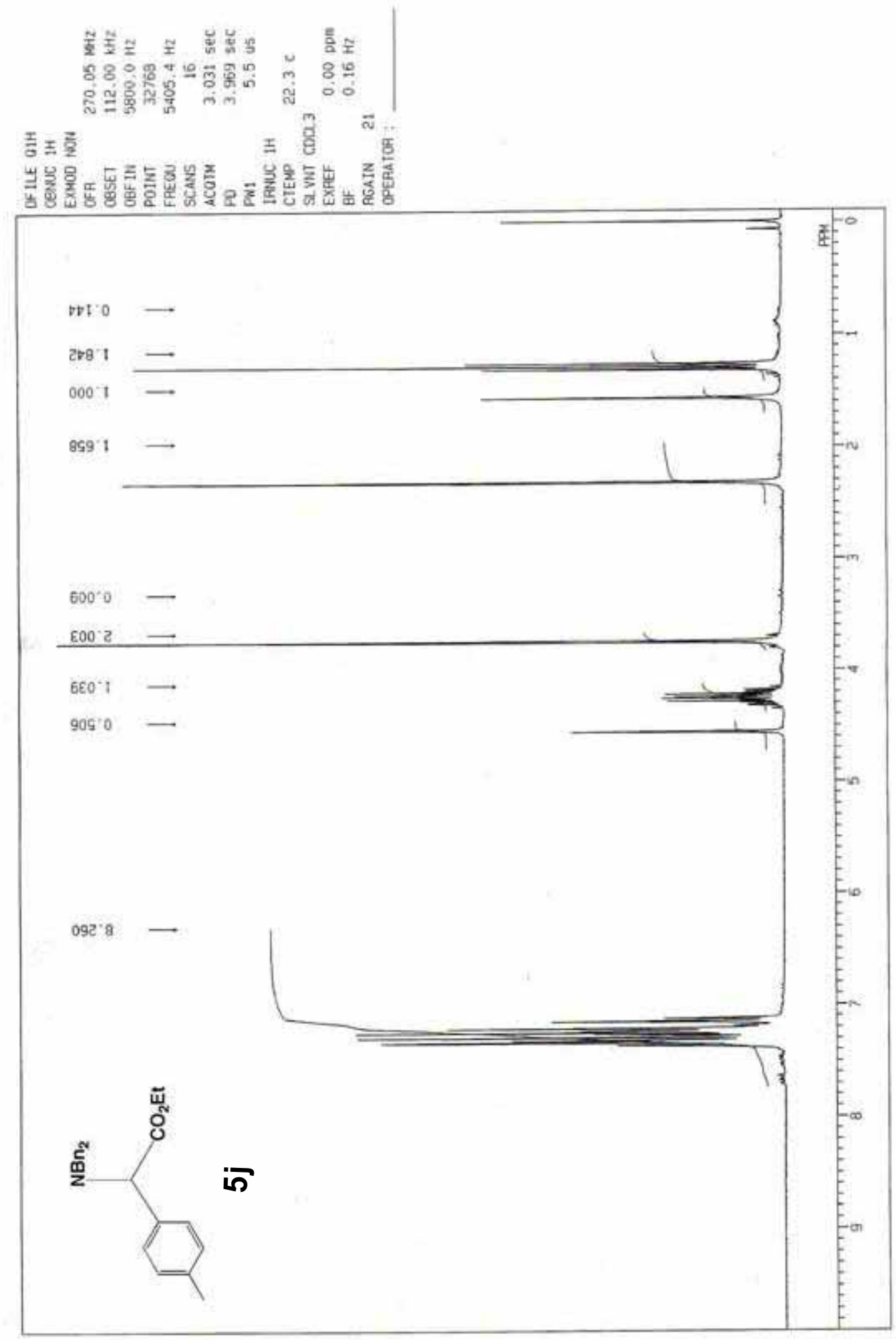




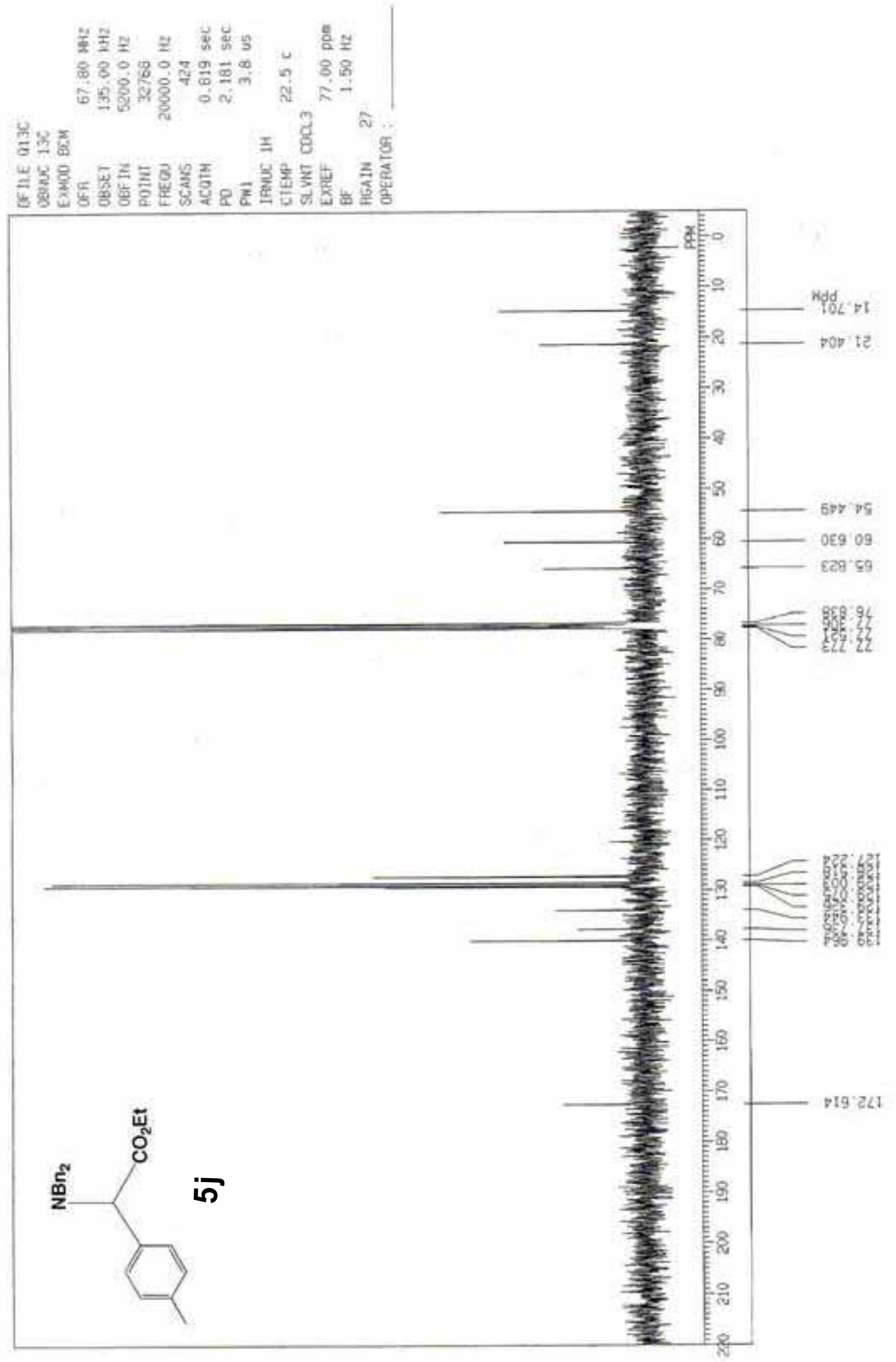




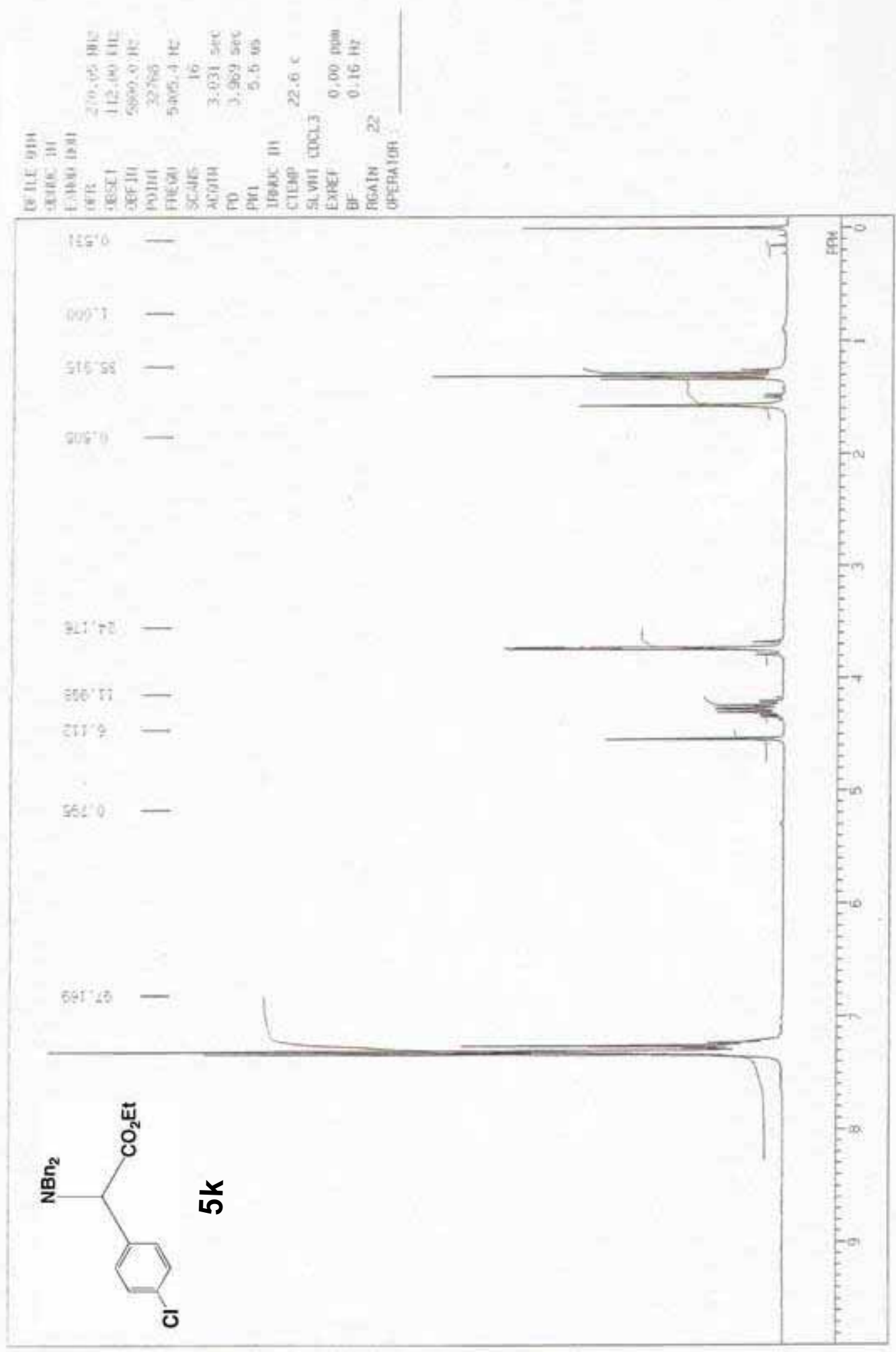




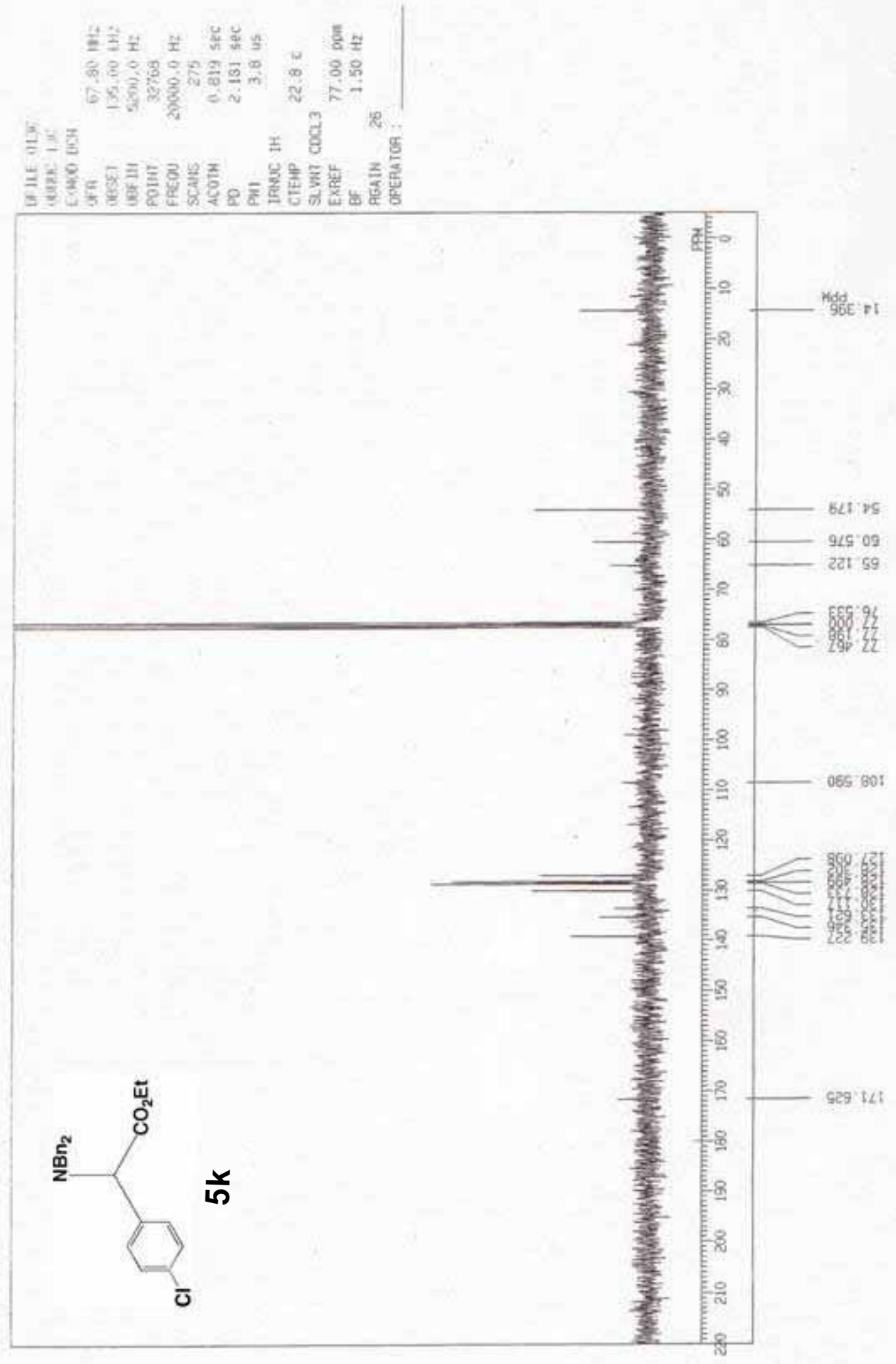



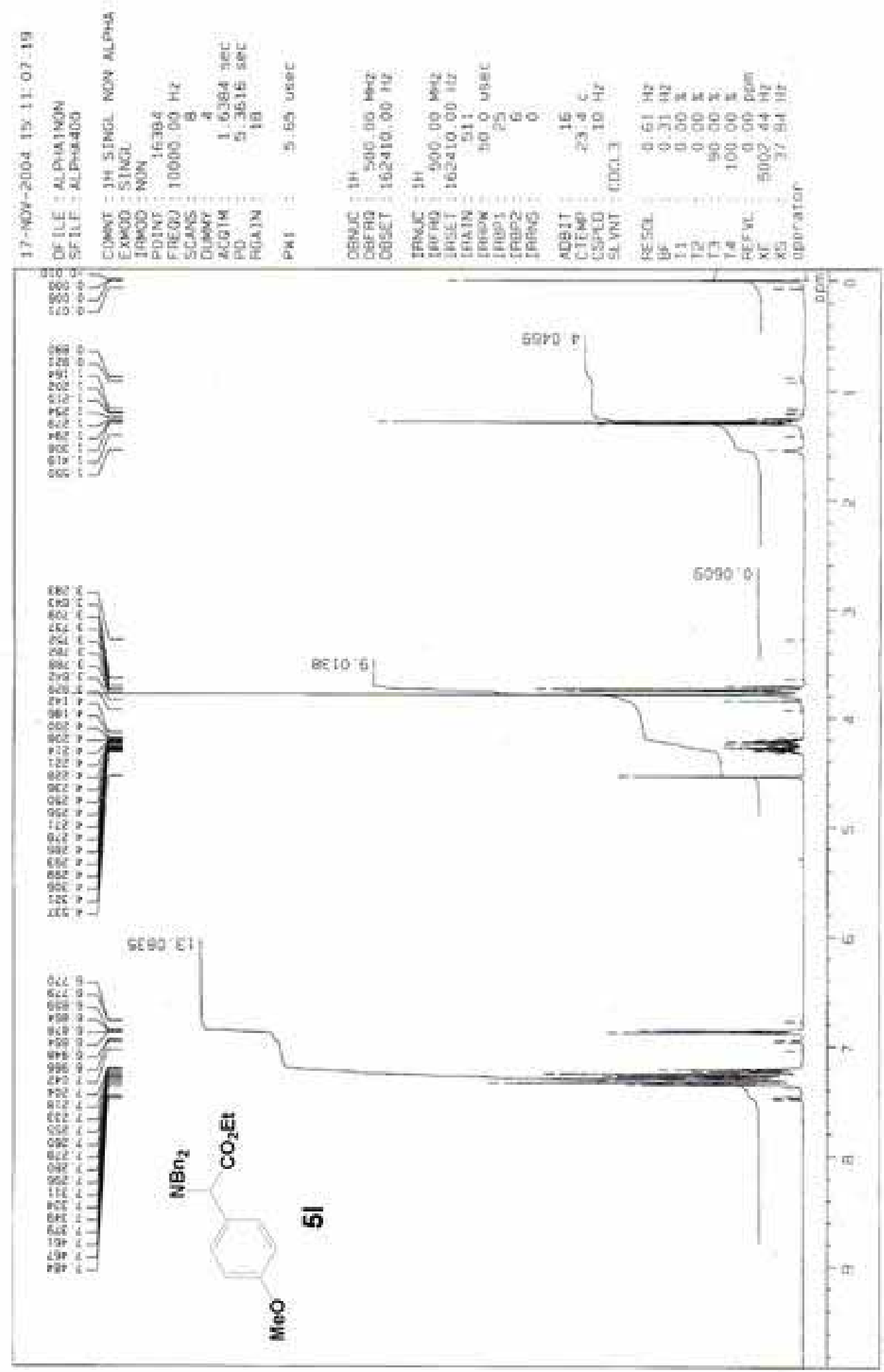


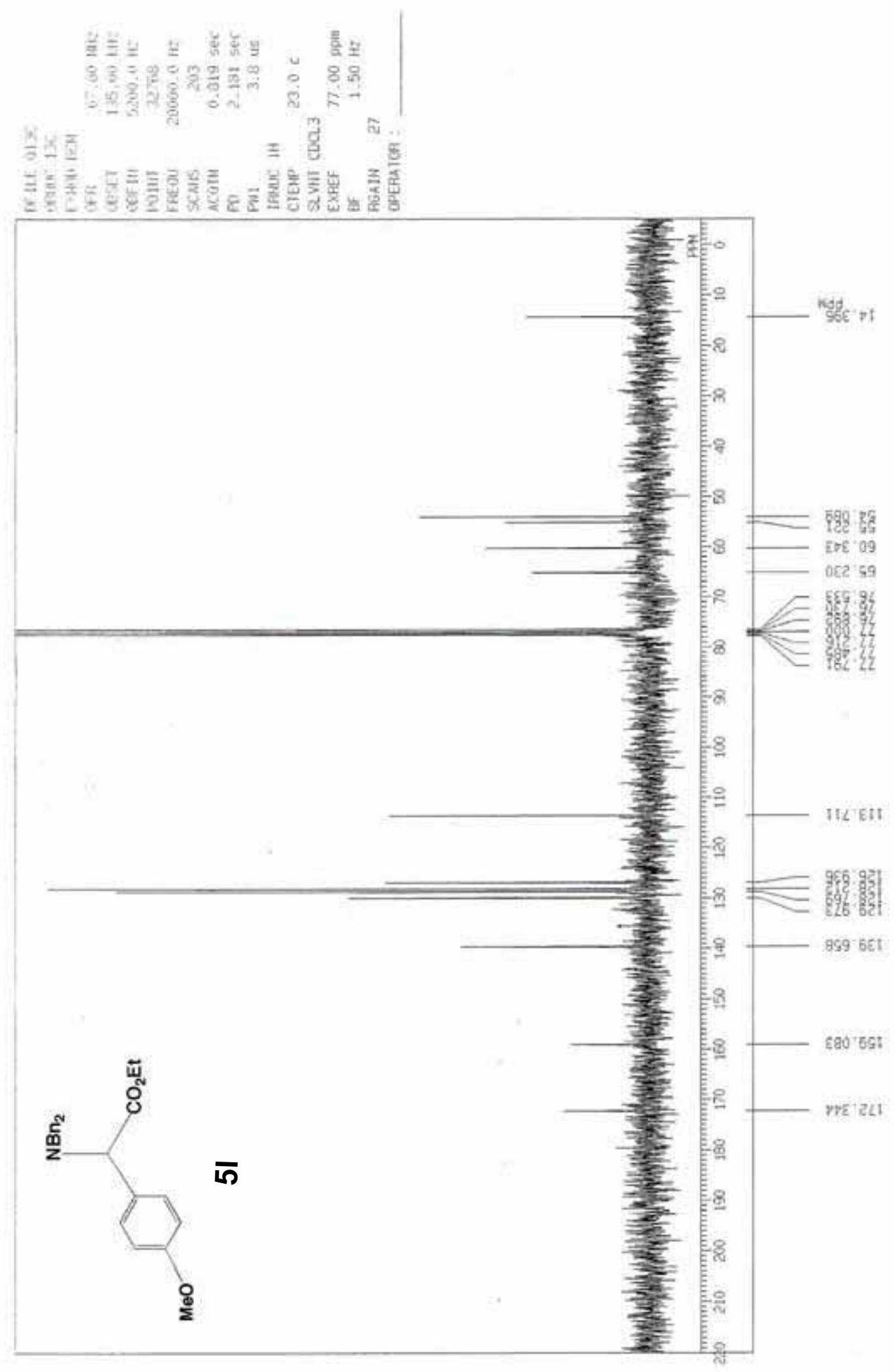




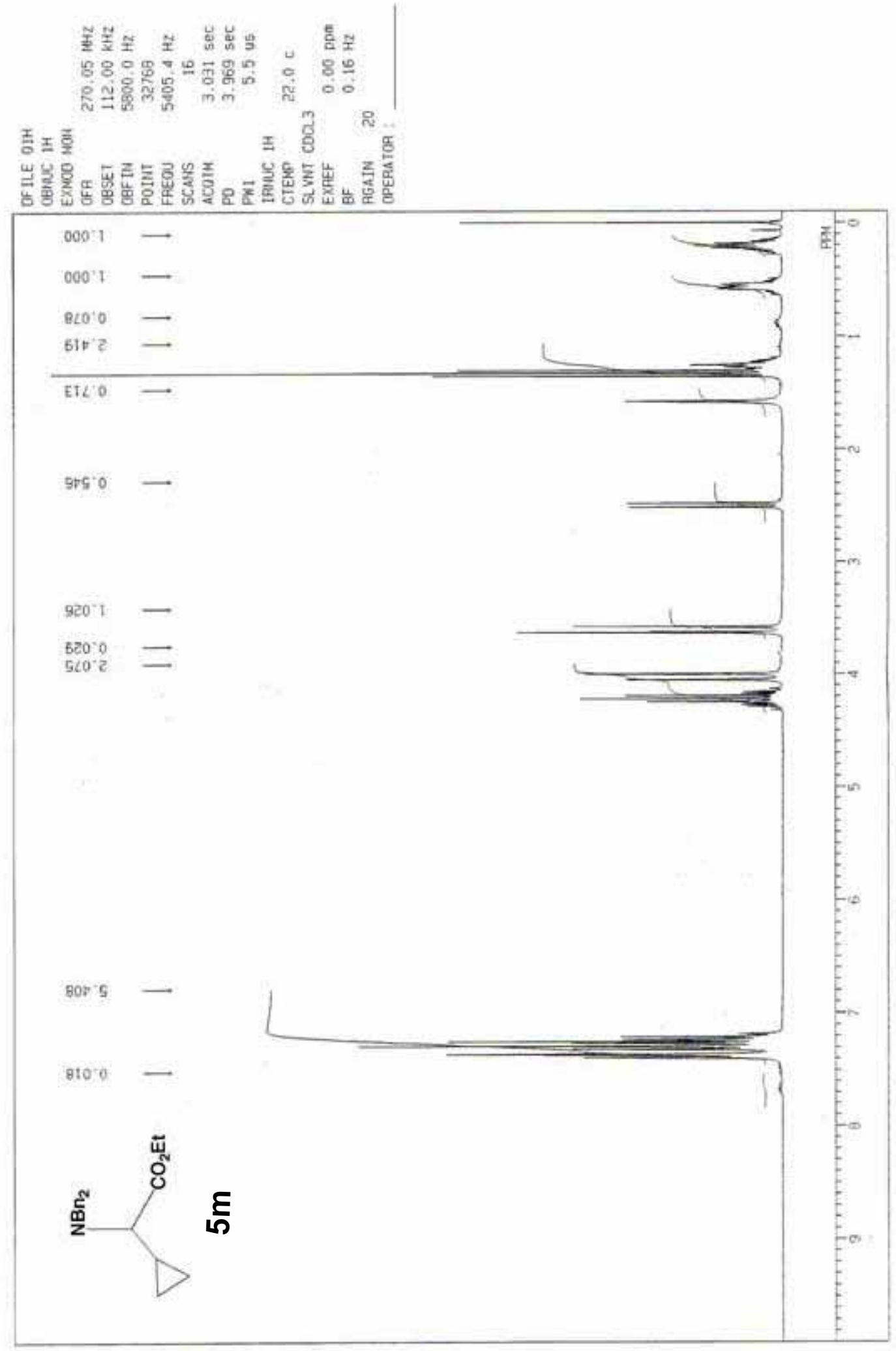




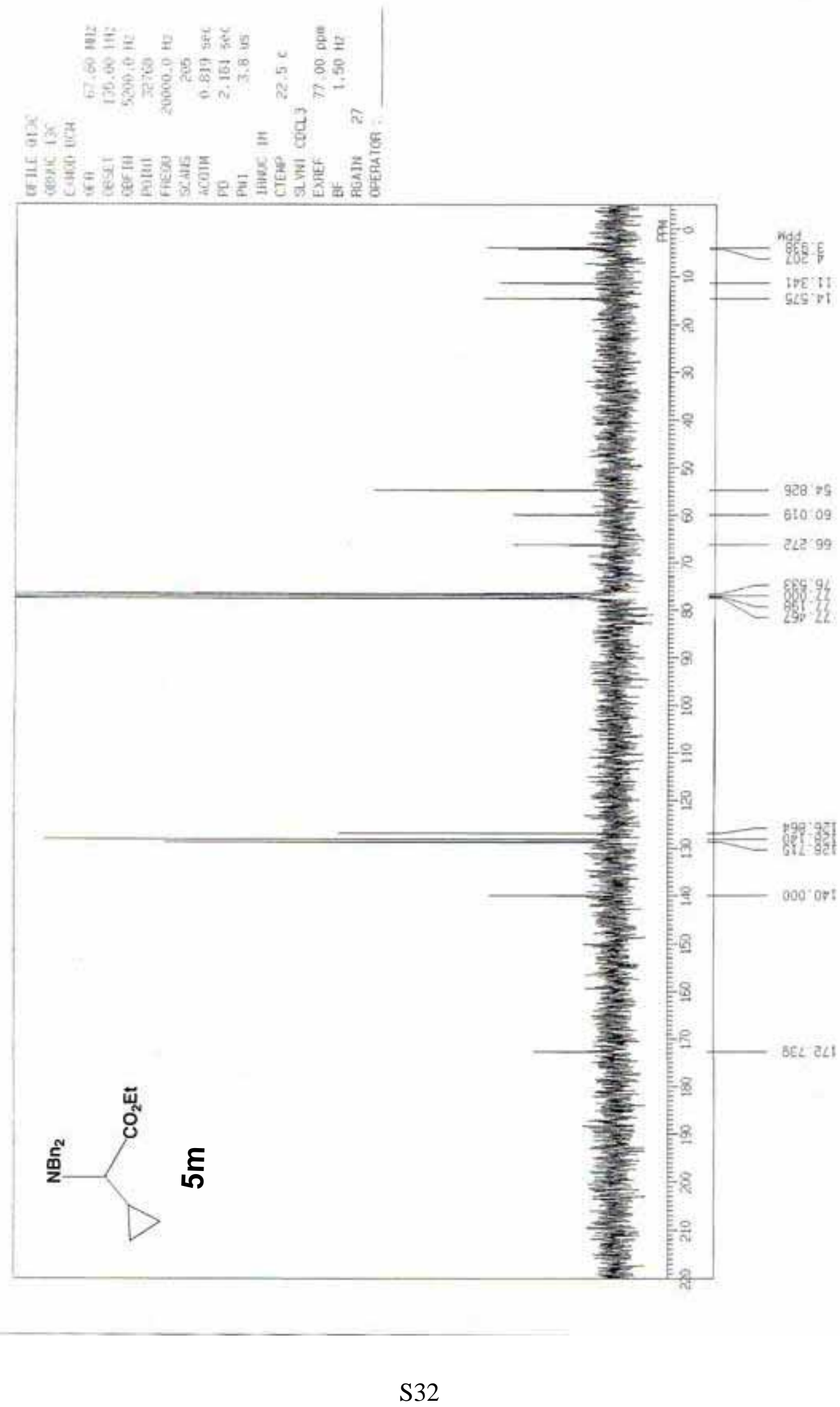




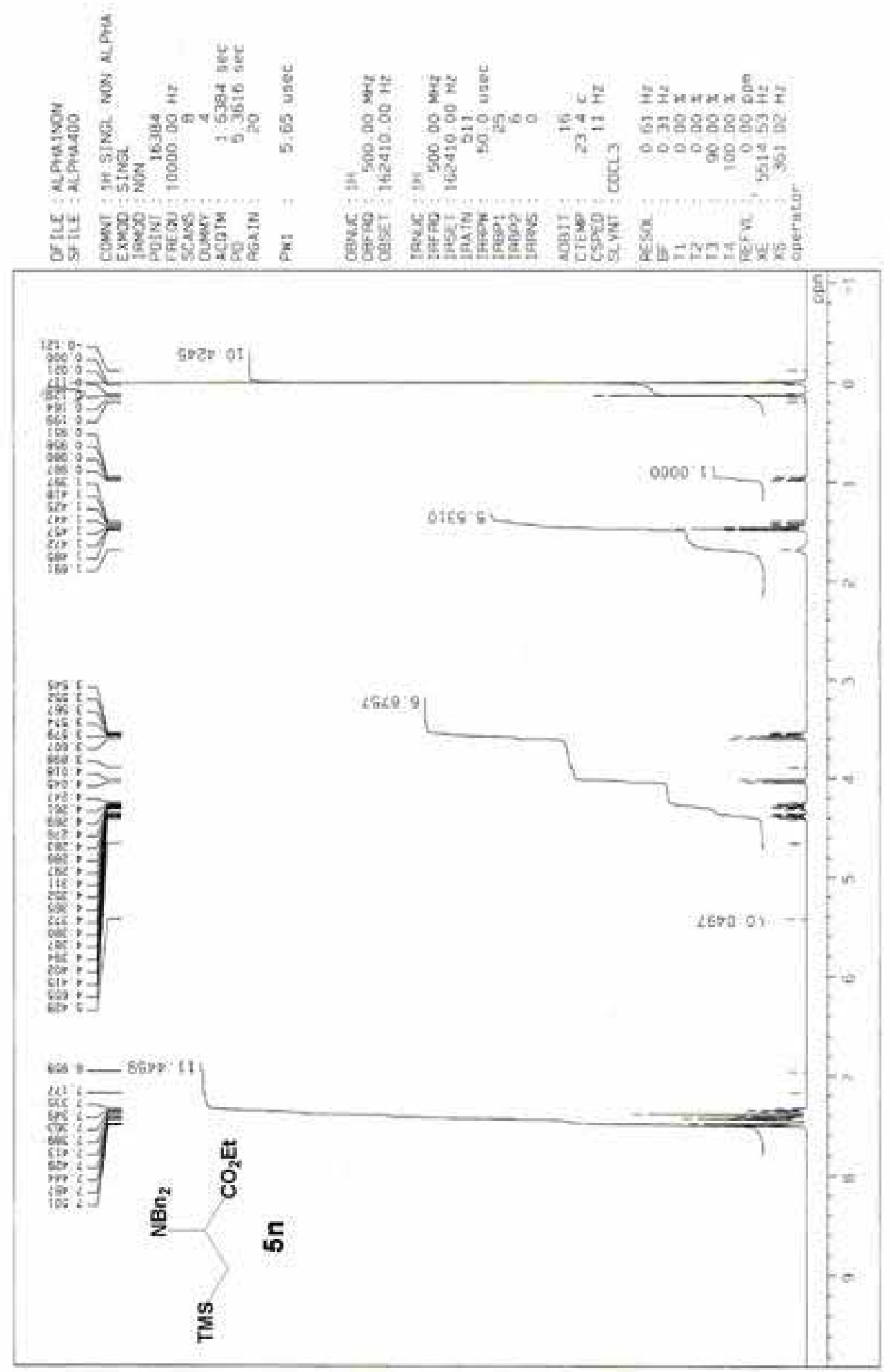




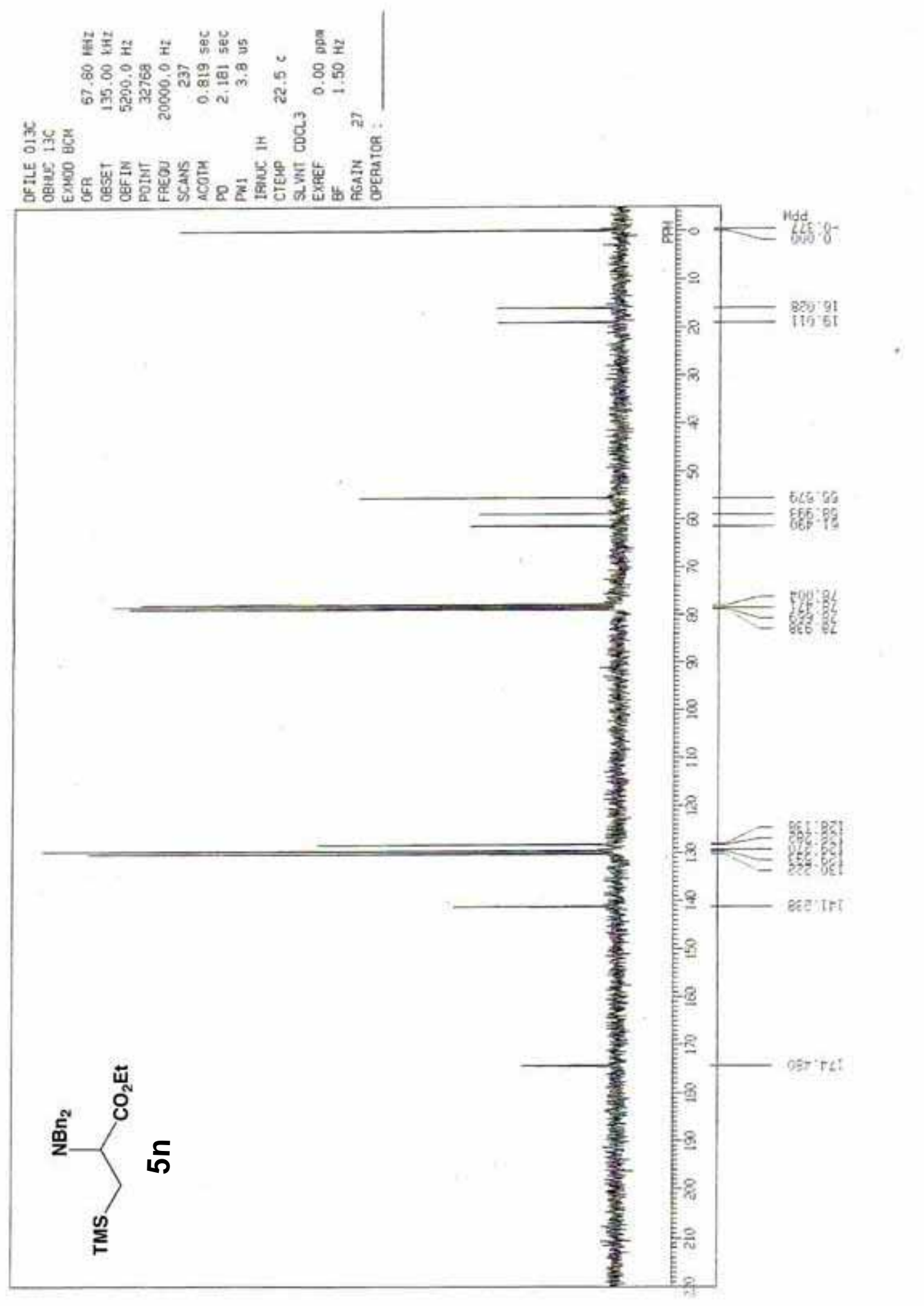




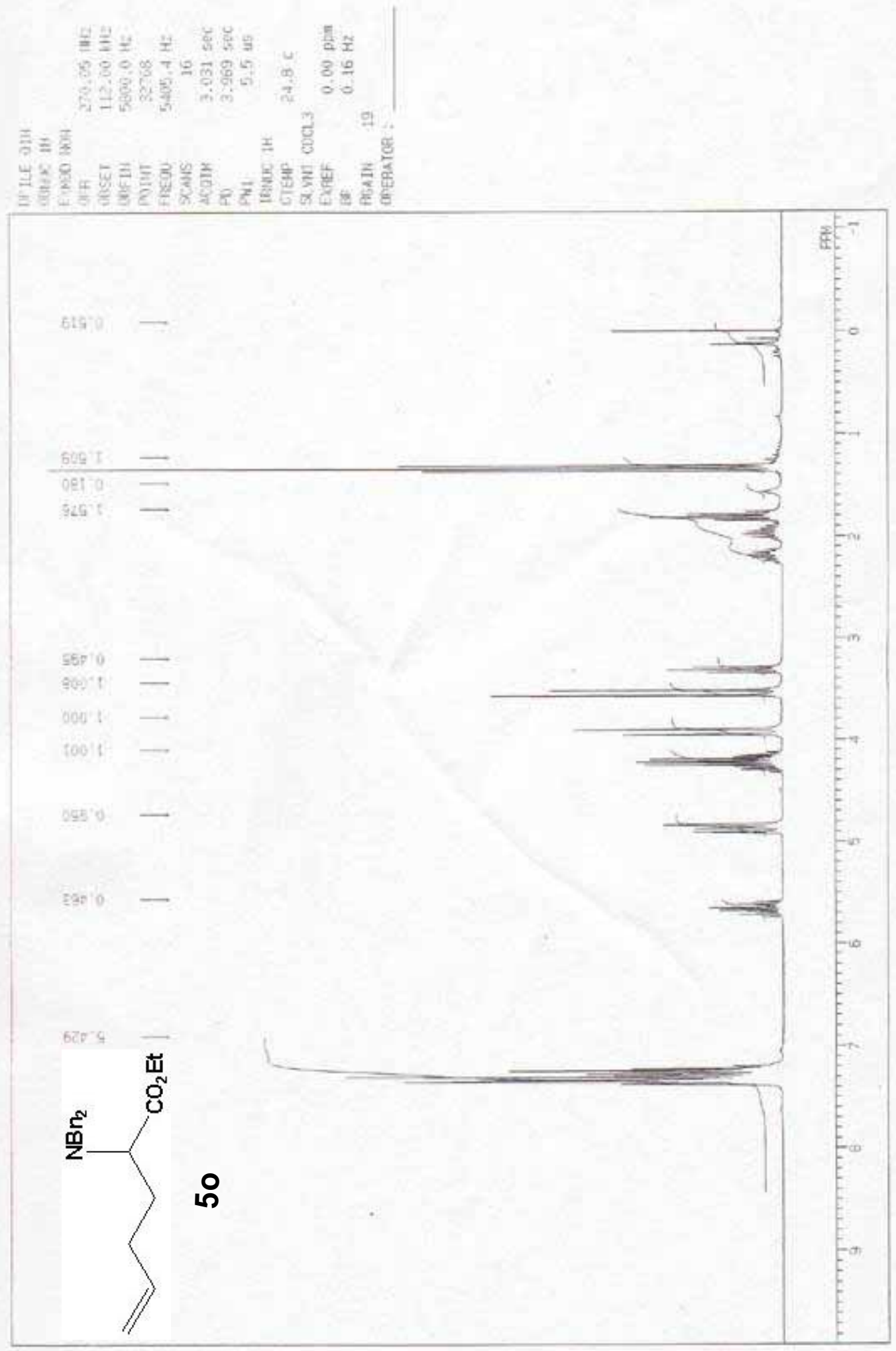




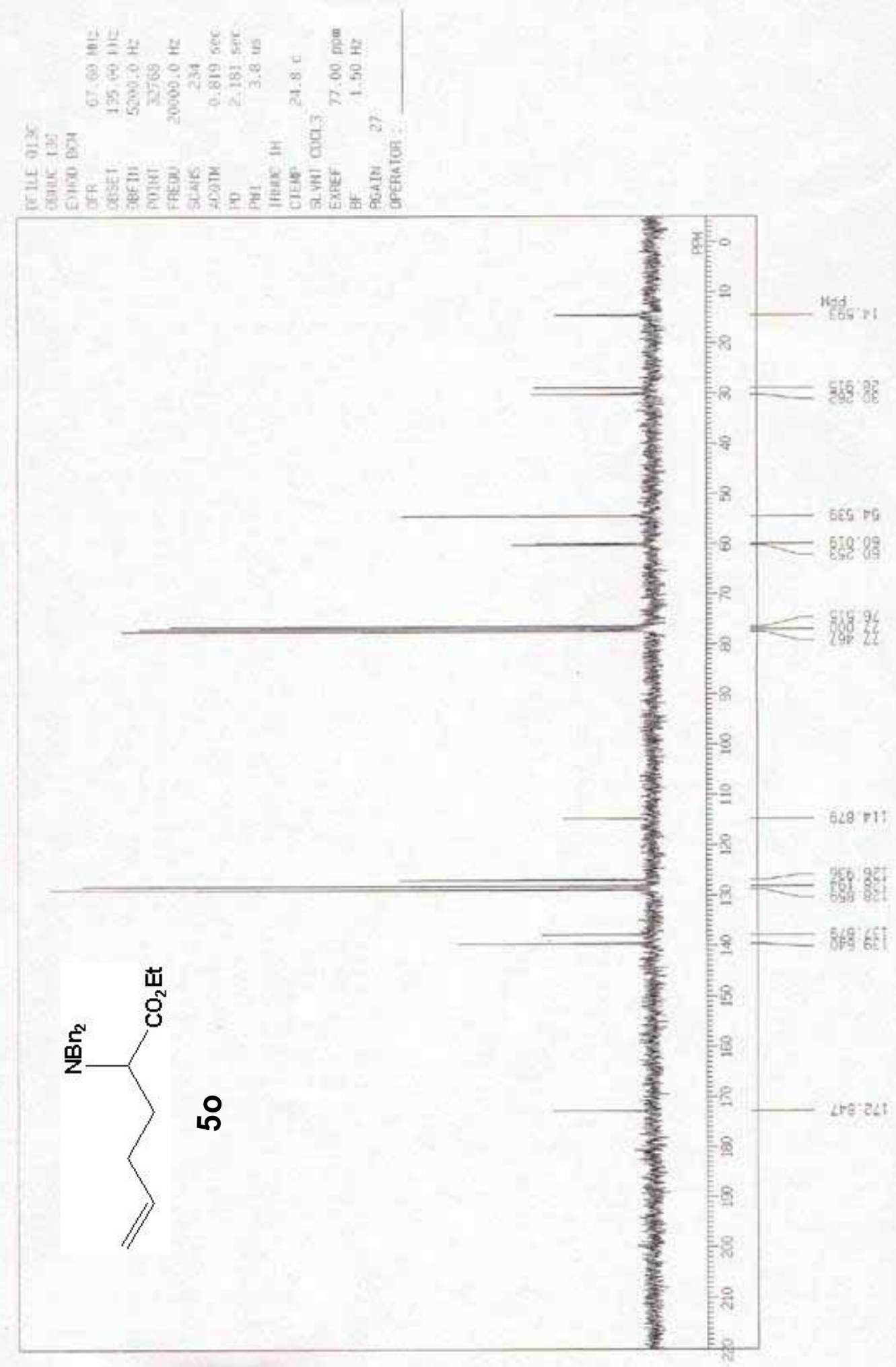




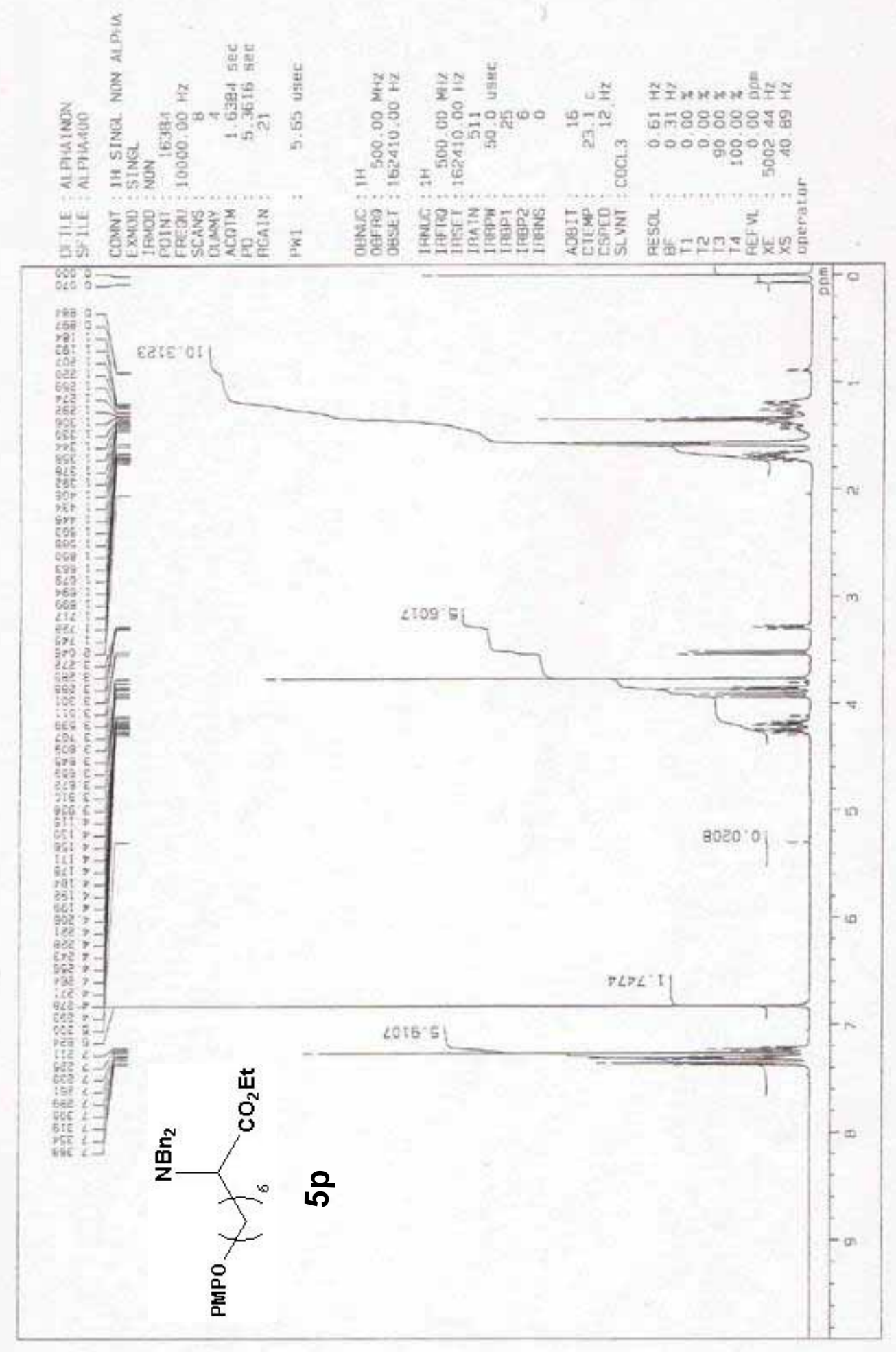




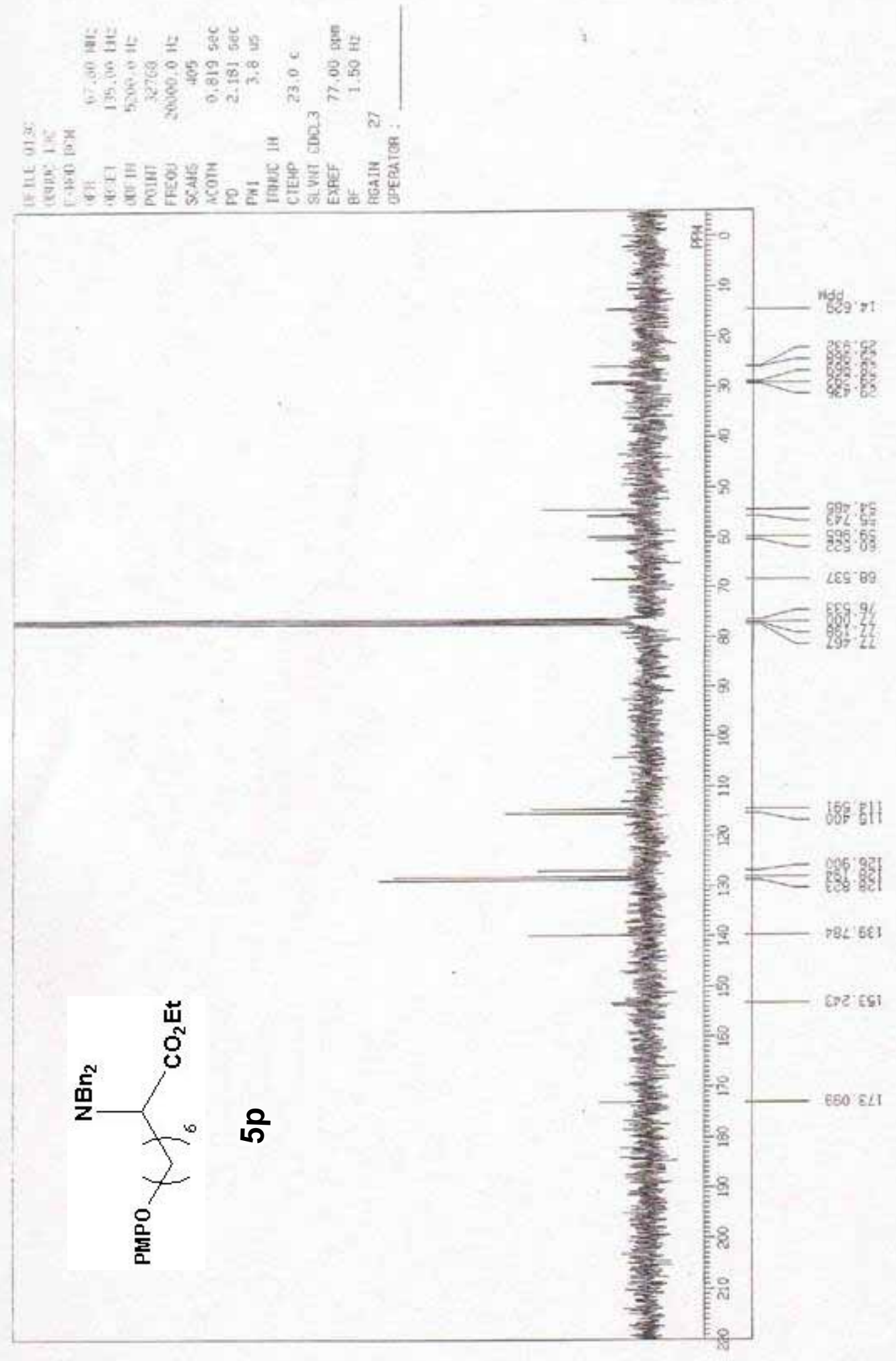




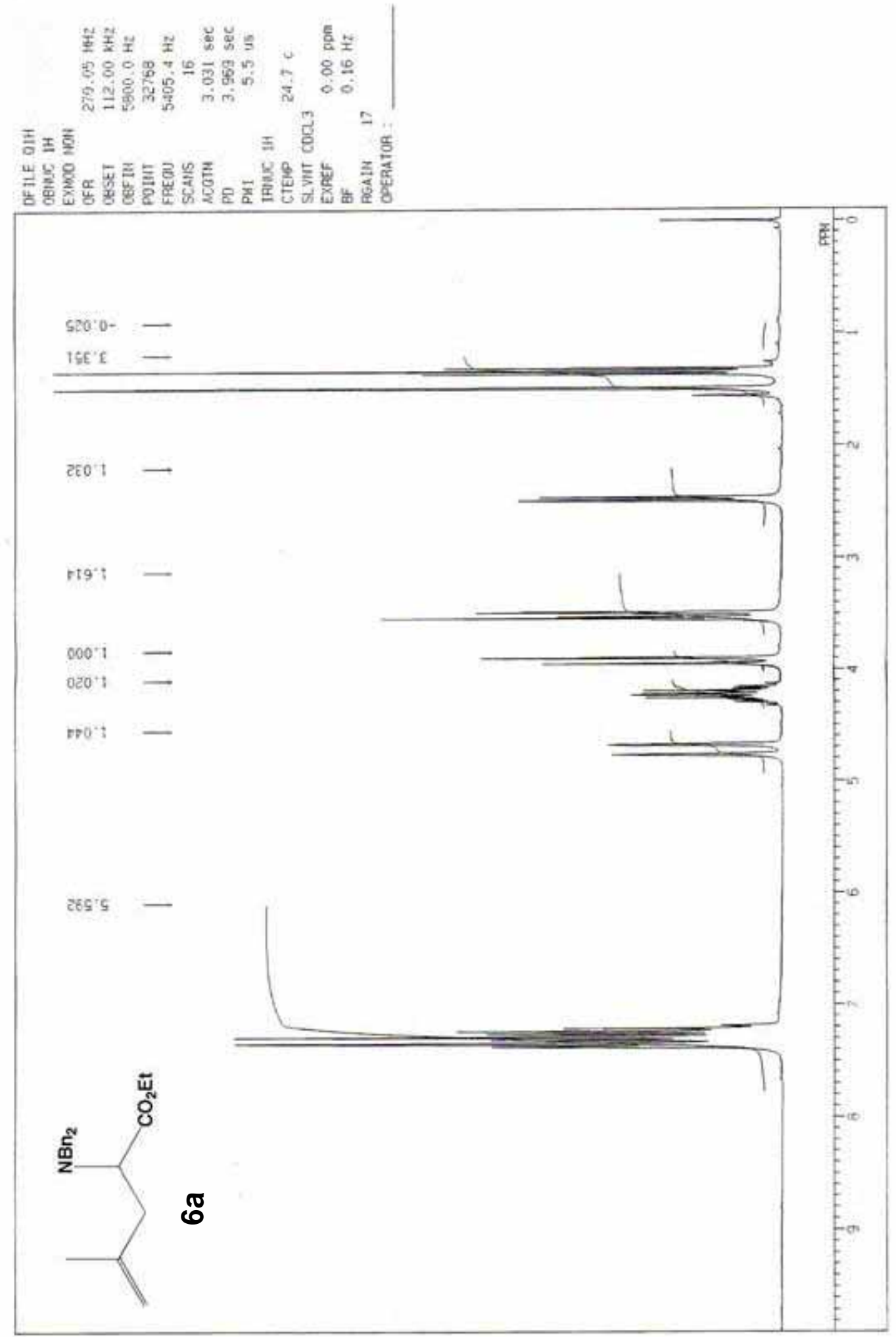




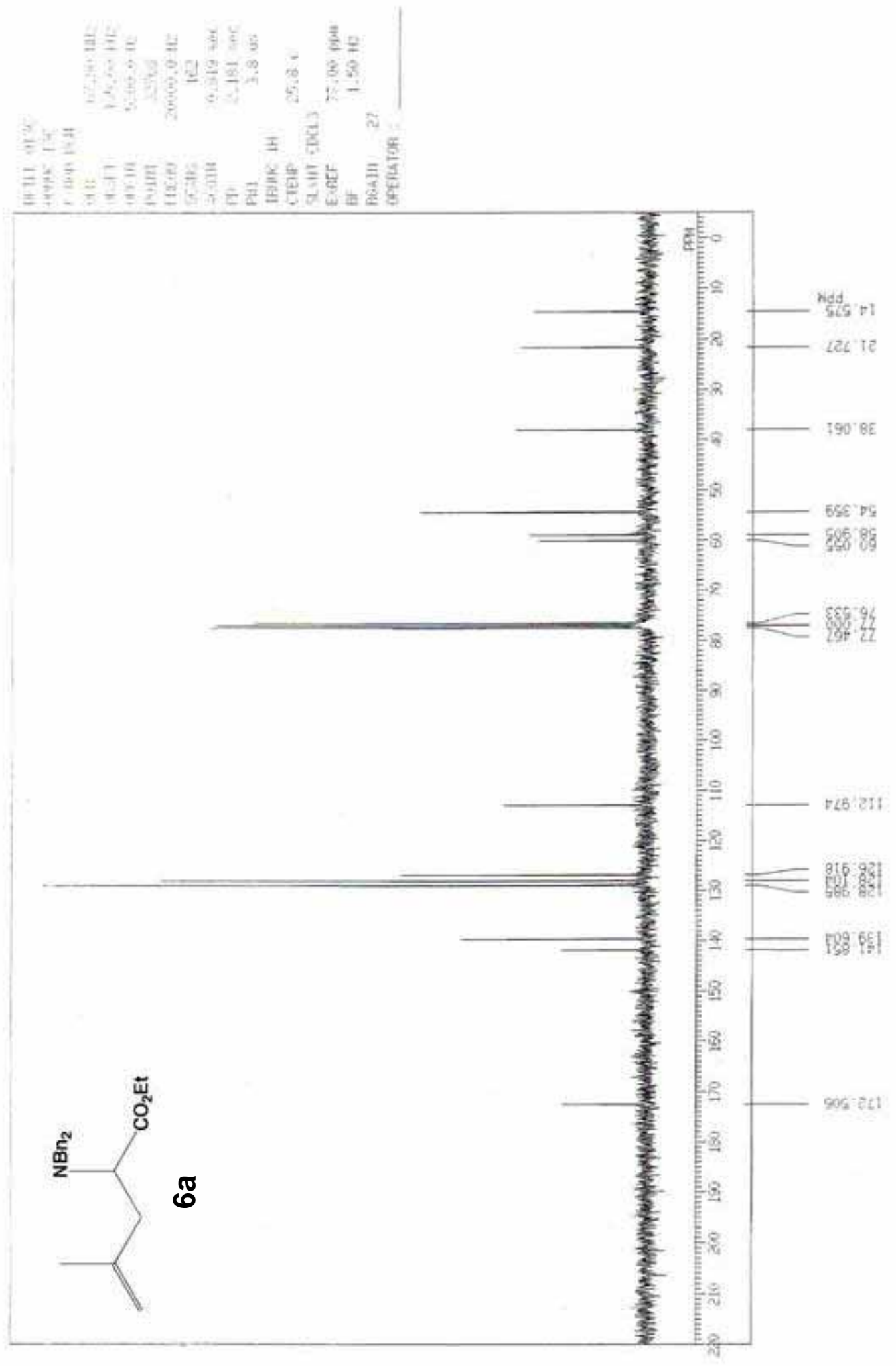




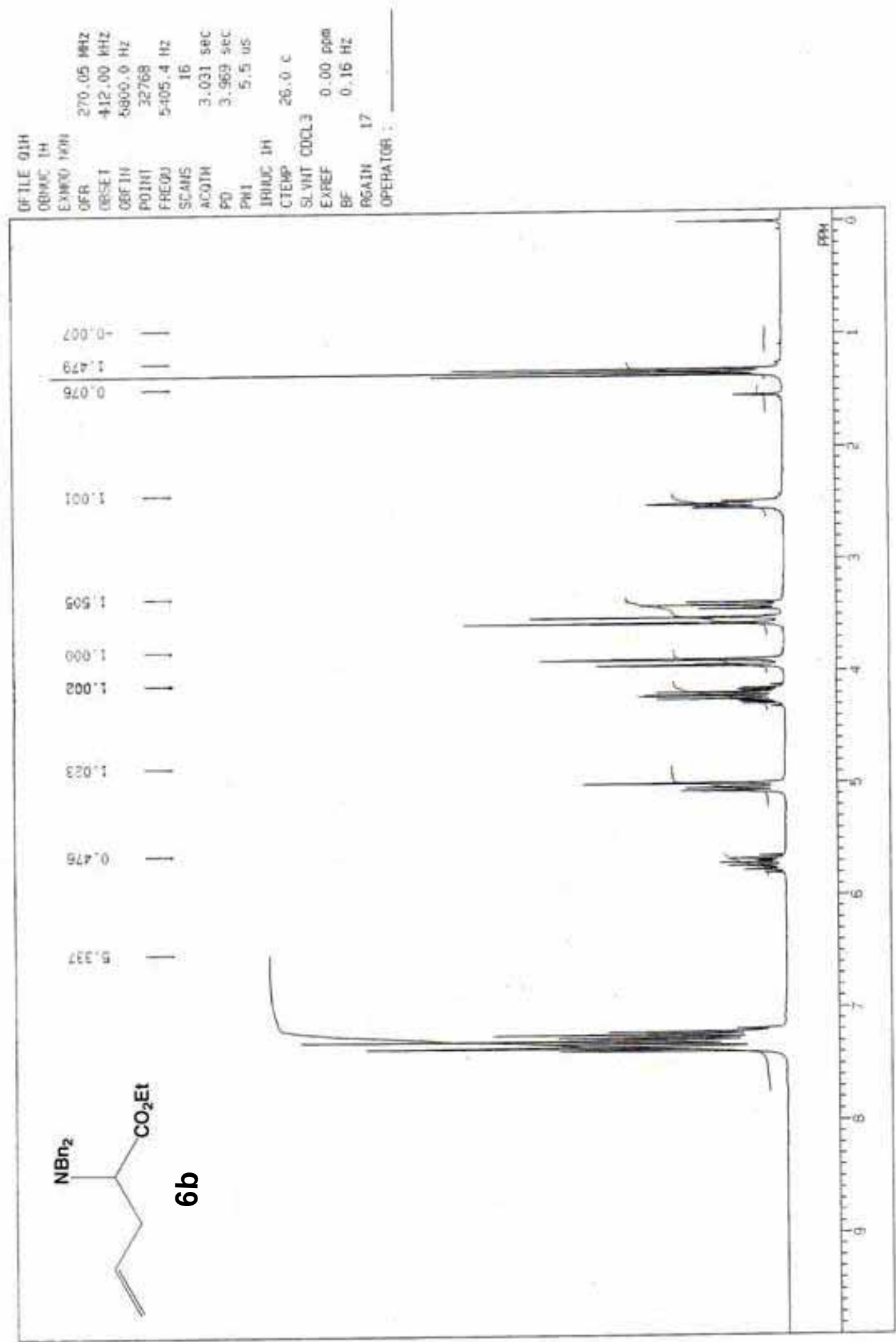




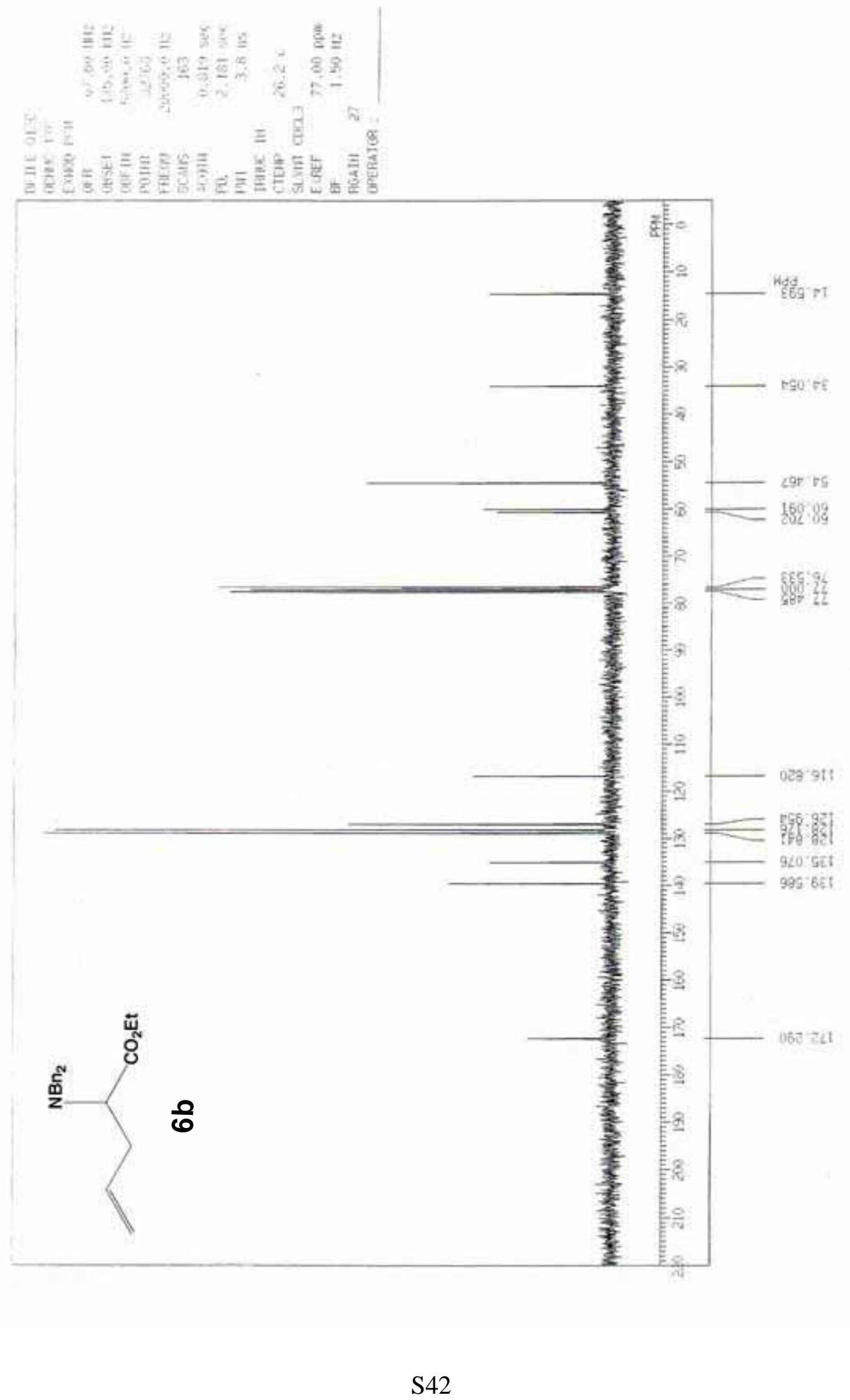



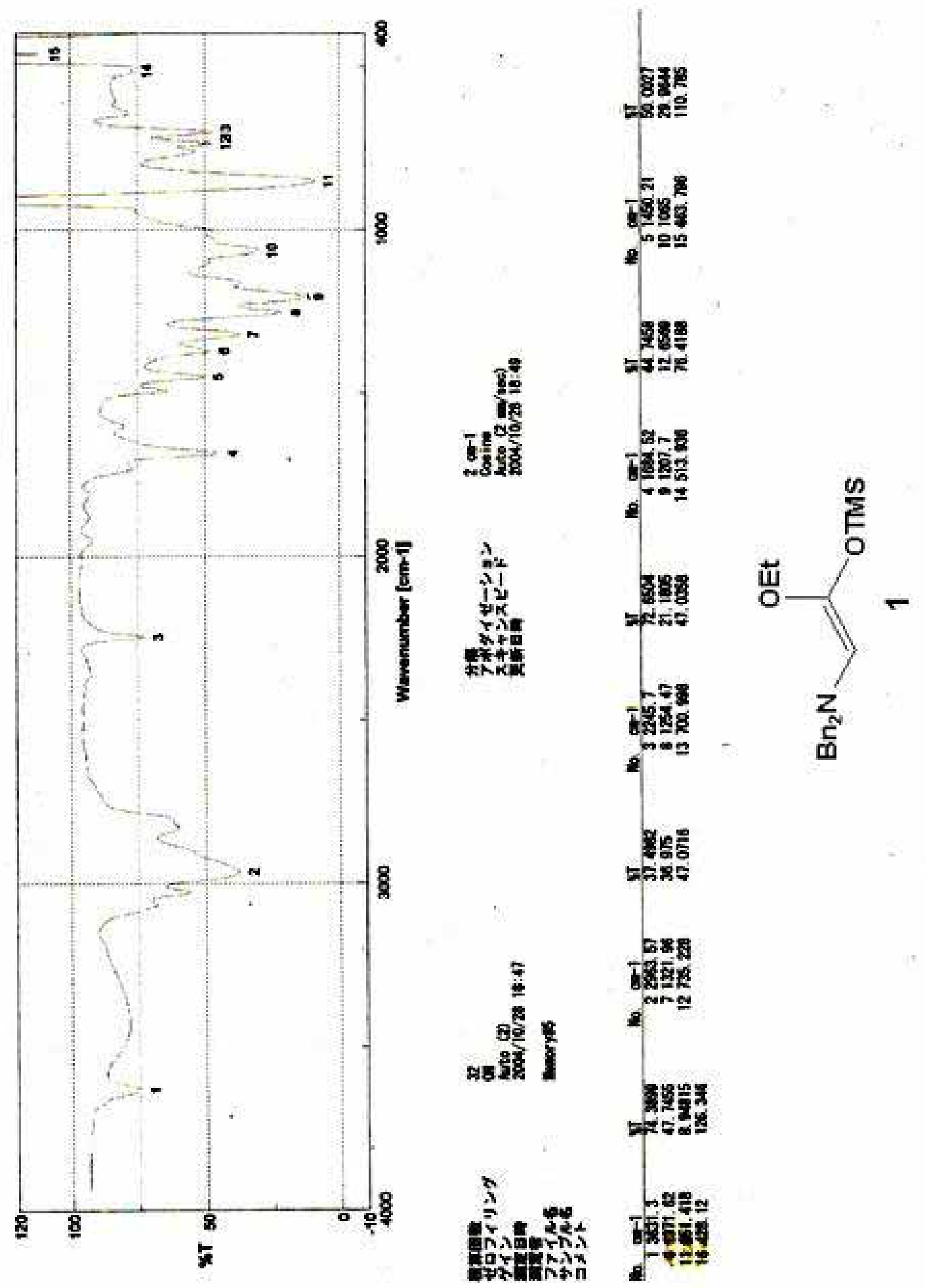


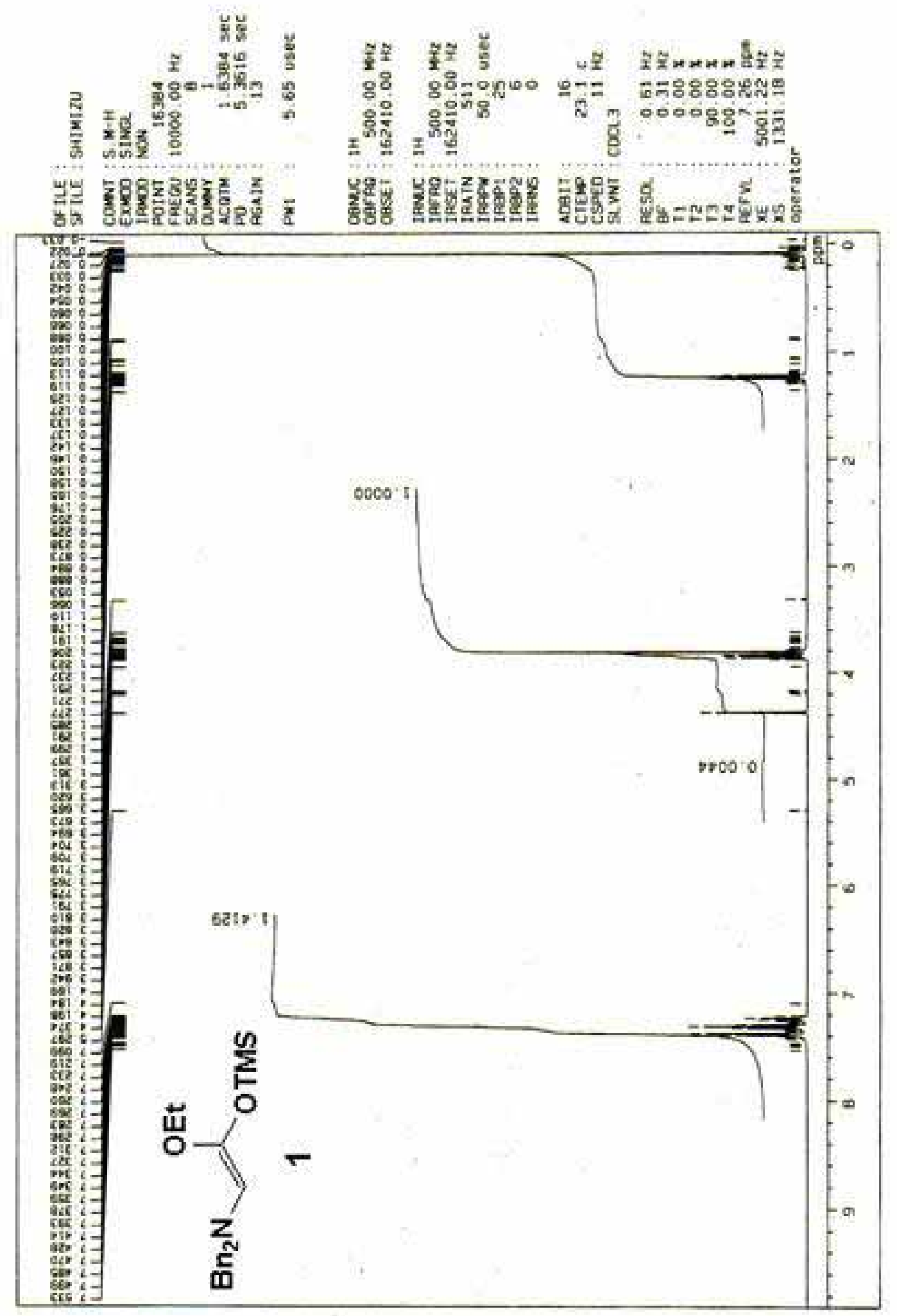




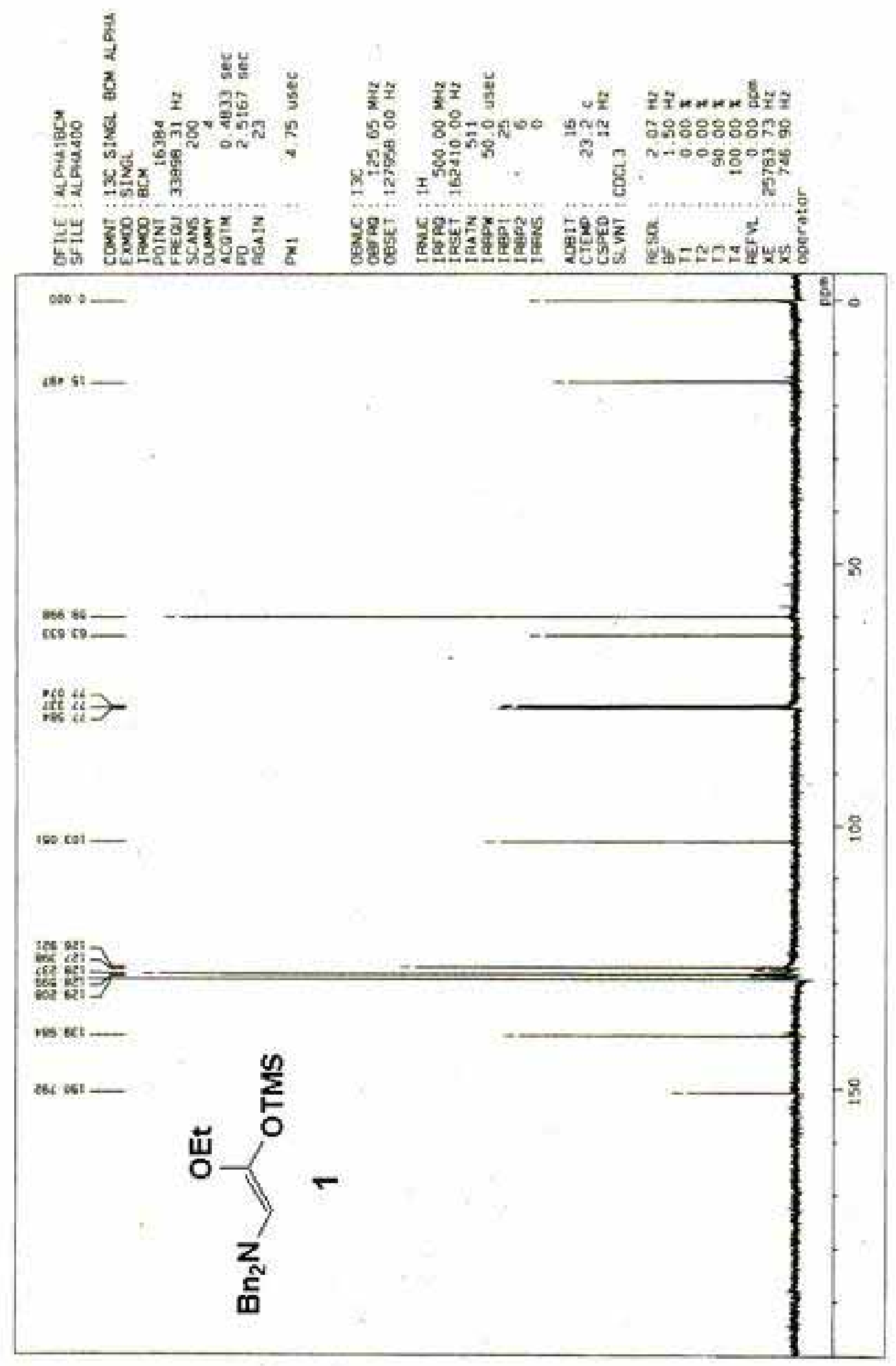




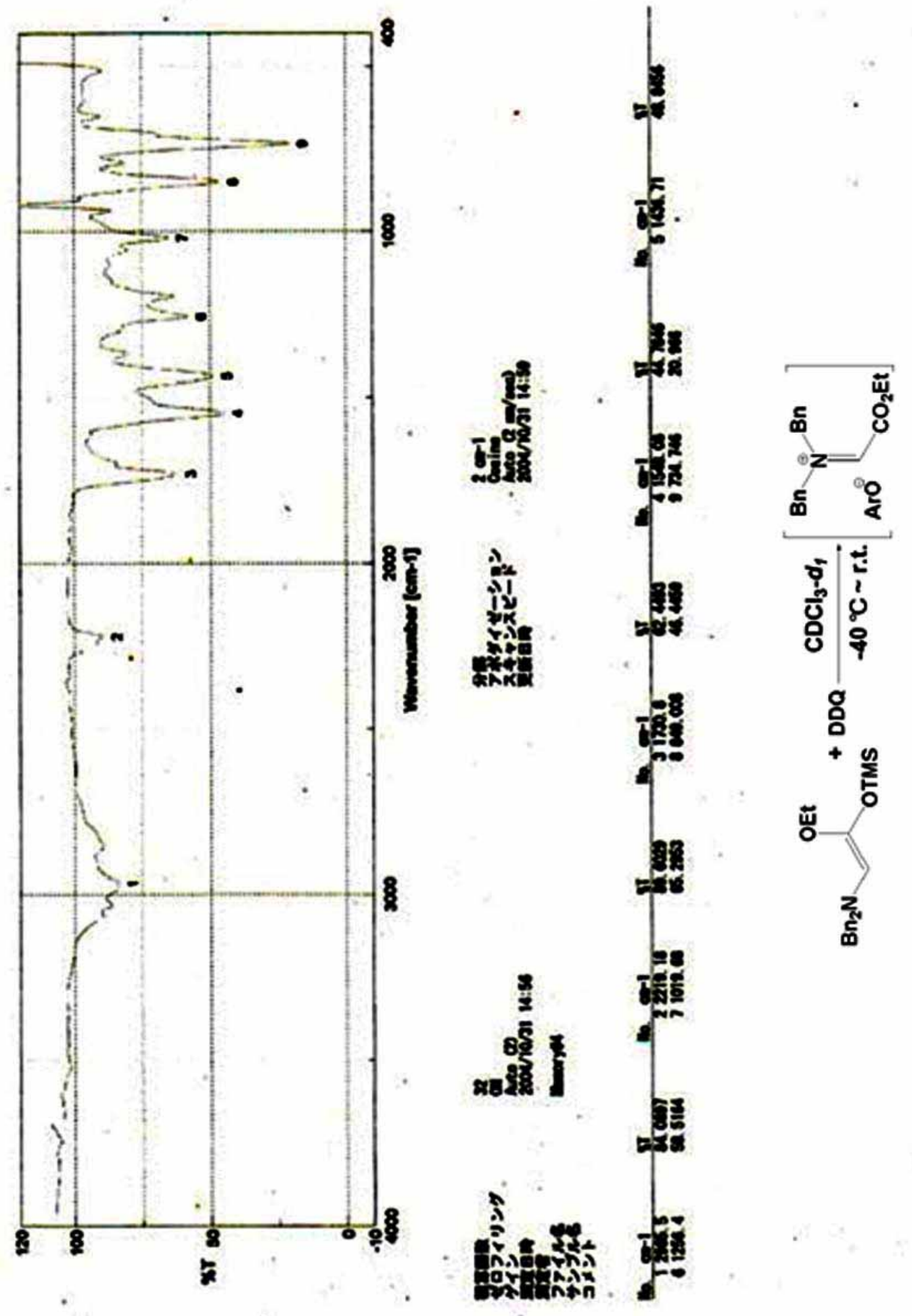




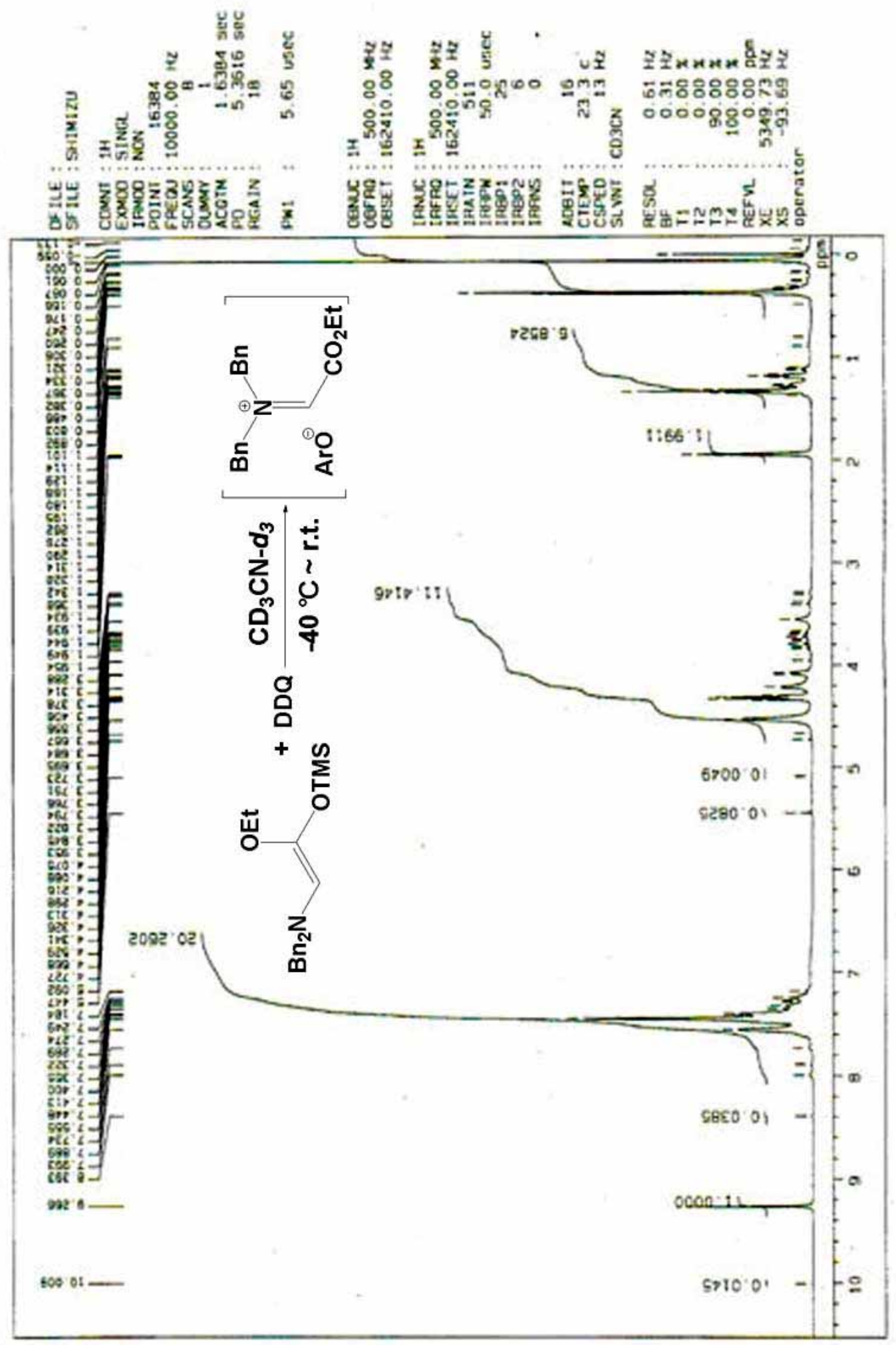




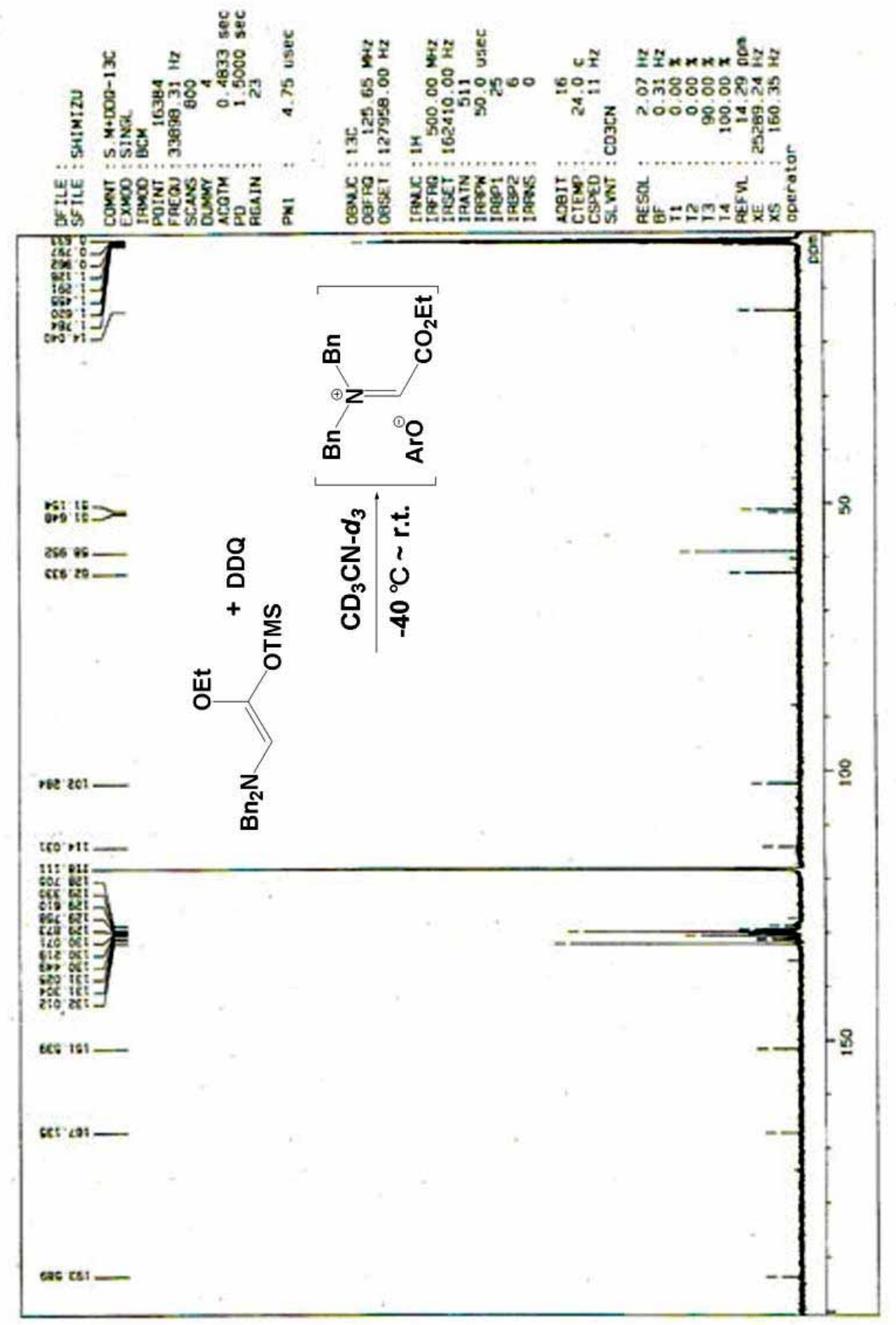

